\title{
Lattice Boltzmann advection-diffusion model for conjugate heat transfer in heterogeneous media
}

\author{
S.A. Hosseini ${ }^{\mathrm{a}, \mathrm{b}, \mathrm{c}, *}$, N. Darabiha ${ }^{\mathrm{b}}$, D. Thévenin ${ }^{\mathrm{a}}$ \\ ${ }^{a}$ Laboratory of Fluid Dynamics and Technical Flows, University of Magdeburg "Otto von Guericke", D-39106 Magdeburg, Germany \\ ${ }^{b}$ Laboratoire EM2C, CNRS, CentraleSupélec, Université Paris-Saclay, 3 rue Joliot Curie, 91192, Gif-sur-Yvette Cedex, France \\ ${ }^{c}$ International Max Planck Research School (IMPRS) for Advanced Methods in Process and Systems Engineering, Magdeburg, Germany
}

\begin{abstract}
Many practical flow configurations involve energy transfer in fluids, or in solids and fluids with different thermo-physical properties. The classical advection-diffusion lattice Boltzmann (LB) solver admits some errors when dealing with such configurations. Given that the macroscopic equation recovered by this model is only valid in the limit of incompressible flows with constant heat capacities, one would, for example, observe inconsistent fluxes at the interface of a fluid and solid with different densities or specific heat capacities. This inconsistency being second-order in space, it will have nonnegligible effects on the final results. In this work, a modified equilibrium distribution function (EDF) is proposed to overcome these issues. The proposed scheme recovers the correct partial differential equation (PDE) describing energy transfer, as shown by a multi-scale Chapman-Enskog analysis. The performance of the model is checked through a variety of test-cases, involving conjugate heat transfer and variable specific heat capacities in both steady and unsteady configurations. In all cases the obtained results are in excellent agreement with reference data.
\end{abstract}

Keywords: Lattice Boltzmann, Energy transfer, Conjugate heat transfer, Variable properties

2010 MSC: 00-01, 99-00

\section{Introduction}

The lattice Boltzmann numerical scheme -and its predecessor the lattice gas automata- have been around for at least three decades now [1. Although initially developed with the sole purpose of solving the Navier-Stokes (NS) equations, the LBM has since then been extended to many applications ranging from quantum mechanics [2] Schrödinger equations- or electron transport in metals [3] to various complex flows such as multi-phase [4, multispecies [5, 6, 7, 8, and non-isothermal systems [9. Classical LB schemes have been extended to non-isothermal flows through three classes of models, (a) the so-called multi-speed schemes [10, 11] and (b) double distribution function (DDF) approaches [12, 13, 14] and (c) Hybrid LB solvers 15, 16]. While the first category can be perceived as a solver for the Boltzmann equation, the other two approaches are direct solvers for the macroscopic energy balance equation. The first class is a natural extension of the basic weakly compressible LB scheme in the context of the kinetic theory, as the number of discrete velocities in the stencil is extended in order to guarantee conservation of internal energy and correctly recover higher-order moments involved in the transport of this conserved variable. Coupling of the bare multi-speed model with the BhatnagarGross-Krook (BGK) collision operator inevitably leads to

\footnotetext{
* Corresponding author

Email address: seyed.hosseini@ovgu.de (S.A. Hosseini)
}

a physical limitation: fixed Prandtl number. This limitation can be dealt with by applying different relaxation coefficients to different moments of the distribution function [17. In practice this can be achieved through the socalled Multiple Relaxation Time (MRT) approach. Apart from this issue, the multi-speed models have been shown to exhibit numerical instabilities and have rather limited stability domains as compared to the classical LB. The stability issue associated to this approach has been dealt with to some extent by the Entropic lattice Boltzmann model (ELBM) 18, 19, 20. The third approach, on the other hand, consists of an LB solver for the flow field coupled to a classical solver (Finite Difference, Finite Volume, Finite Element, Spectral) for the energy field. While allowing for a straightforward treatment of complex terms in the energy balance equation, a stable way of coupling both solvers is still challenging given the different stability thresholds of the different numerical solvers.

The DDF models constitute another class of approach for simulating non-isothermal flows. As suggested by its name, in this approach the flow field is modeled by the classical LB solver, while another set of distribution functions is used to model temperature. The flow and temperature fields are coupled through the advection velocity and possibly additional force terms - in the context of the Boussinesq approximation for example. This class of approach shows advantages in terms of simplicity, locality, and stability over the multi-speed models. In terms of 
computation and memory consumption, DDF models are also more advantageous as they deal with a smaller number of discrete velocities, leading to more local time-evolution operations, in turn yielding a better parallel performance. However, DDF models suffer from a number of shortcomings stemming from the simple physics involved.

One major issue with the classical advection-diffusion LB solver for temperature is that of flux mismatch at interfaces. Considering only diffusive scaling for the sake of this introductory discussion, the first-order non-equilibrium flux, $\mathbf{q}^{(1)}$, recovered by this approach at macroscopic scale is:

$$
\mathbf{q}^{(1)}=\frac{\lambda}{\rho c_{p}} \nabla T,
$$

where $\lambda$ is the heat diffusion coefficient. This flux is only correct for a constant specific heat capacity $c_{p}$ and density $\rho$ over the simulation domain 21. One approach to

5 alleviate this restriction is to include an appropriate correction term at interfaces. This approach has been successfully applied to conjugate heat transfer in heterogeneous media 22, 23, 24, 25, 26. The main shortcomings of this approach are practicality and limited applicability to more general cases. For cases involving numerous interfaces, such as composite materials and porous media, applying a correction term at all interface nodes is not an efficient solution. Furthermore, this solution only deals with discontinuities in thermodynamic parameters at interfaces 15 and cannot model flows with temperature- or compositiondependent specific heat capacity for example. In the past couple of years various LB schemes have been developed to model heat transfer with variable specific heat capacity for specific applications such as porous media [27, 28].

${ }_{20}$ The aim of the present work is to develop an advectiondiffusion LB model both efficient, easy to implement, and extensible to any type of flow with any given degree of simplification, up to full models. In the next section, the classical advection-diffusion LB model will be presented 25 along with its shortcomings. The second and third sections will then focus on introducing the proposed model and validating it through a number of standard test-cases.

\section{Energy balance and the Classical AD-LB ap- proach to modeling energy transport}

At the macroscopic scale, energy balance in the system can be formulated through transport equations for a variety of quantities, e.g. internal energy, total energy, enthalpy etc. A thorough review of the different formulation can be found in 29. For example the balance equation for sensible enthalpy can be written as:

$$
\partial_{t} \rho h_{s}+\nabla \cdot \rho h_{s} \mathbf{u}=\dot{\omega}_{T}+\partial_{t} p+\mathbf{u} \cdot \nabla p+\nabla \cdot \lambda \nabla T,
$$

where $h_{s}$ is the sensible enthalpy, $\dot{\omega}_{T}$ is the heat production rate (for instance due to chemical reactions), $T$ is the temperature and $p$ is pressure. Here for the sake of simplicity, terms such as viscous dissipation, enthalpy transport by species diffusion etc have been ignored.

The classical approach to solving the heat transport equation in LB consists of a time evolution equation for the temperature distribution function, denoted $h_{\alpha}$ here, similar to the flow field:

$$
\begin{aligned}
& h_{\alpha}\left(\mathbf{x}+\mathbf{c}_{\alpha} \delta_{t}, t+\delta_{t}\right)-h_{\alpha}(\mathbf{x}, t)= \\
& \frac{1}{\tau_{T}}\left(h_{\alpha}^{(e q)}(\mathbf{x}, t)-h_{\alpha}(\mathbf{x}, t)\right)+w_{\alpha} \frac{\dot{\omega}_{T}(\mathbf{x}, t) \delta_{t}}{\rho_{0} c_{p, 0}}+w_{\alpha} \mathbf{c}_{\alpha} \cdot \mathbf{F},
\end{aligned}
$$

where $\mathbf{F}$ is a forcing term -explained later on in this subsection, $\rho_{0}$ is the fluid density, $c_{p, 0}$ is the specific heat capacity (the subscript 0 is used here as this model is only adapted to incompressible flows with constant heat capacity), $h_{\alpha}^{(e q)}$ is the equilibrium distribution function, $\delta_{t}$ is the time-step size, $c_{s}$ is the so-called "lattice sound speed", $\mathbf{c}_{\alpha}$ is the velocity vector associated to each propagation direction $\alpha$ and $w_{\alpha}$ is the corresponding weight fulfilling the following conditions:

$$
\begin{gathered}
\sum_{\alpha} h_{\alpha}^{(e q)}(\mathbf{x}, t)=T(\mathbf{x}, t), \\
\sum_{\alpha} \mathbf{c}_{\alpha} h_{\alpha}^{(e q)}(\mathbf{x}, t)=\mathbf{u}(\mathbf{x}, t) T(\mathbf{x}, t), \\
\sum_{\alpha} \mathbf{c}_{\alpha} \cdot \mathbf{c}_{\alpha} h_{\alpha}^{(e q)}(\mathbf{x}, t)=\mathrm{D} c_{s}^{2} T(\mathbf{x}, t),
\end{gathered}
$$

where $\mathrm{D}$ is the physical dimension of the simulation. The EDF can be defined as:

$$
h_{\alpha}^{(e q)}(\mathbf{x}, t)=w_{\alpha} T(\mathbf{x}, t)\left(1+\frac{1}{c_{s}^{2}} \mathbf{c}_{\alpha} \cdot \mathbf{u}(\mathbf{x}, t)\right),
$$

where $T(\mathbf{x}, t)$ is the local temperature, and $\mathbf{u}(\mathbf{x}, t)$ is the local fluid velocity. For the linear EDF, Eq. 6 can also be expressed as:

$$
\sum_{\alpha} \mathbf{c}_{\alpha} \otimes \mathbf{c}_{\alpha} h_{\alpha}^{(e q)}(\mathbf{x}, t)=c_{s}^{2} T(\mathbf{x}, t) \mathbf{I}
$$

where $\mathbf{I}$ is the identity matrix and $\otimes$ is the Kronecker product. It is worth mentioning that the EDF can also take a non-linear form - similar to the flow field solver EDF. The relaxation coefficient, $\tau_{T}$ appearing in Eq. 3 is usually defined as:

$$
\tau_{T}=\frac{1}{\delta_{t} c_{s}^{2}} \frac{\lambda}{\rho_{0} c_{p, 0}}+\frac{1}{2} .
$$

Using the Chapman-Enskog multi-scale analysis, the classical model presented in this section -without the force term $\mathbf{F}$ - is shown to recover under convective scaling the following Partial Differential Equation (PDE) up to second order:

$$
\begin{aligned}
\partial_{t} T+\nabla \cdot(\mathbf{u} T)- & \nabla \cdot \frac{\lambda}{\rho_{0} c_{p, 0}} \nabla T \\
& -\nabla \cdot \frac{\lambda}{c_{s}^{2} \rho_{0} c_{p}} \partial_{t} \mathbf{u} T-\frac{\dot{\omega}_{T}}{\rho_{0} c_{p, 0}}=0 .
\end{aligned}
$$


In order to take out the fourth term on the LHS, which is an unphysical term, the force term $\mathbf{F}$ of Eq. 3 must be set to 30]:

$$
\mathbf{F}=\frac{1}{c_{s}^{2}}\left(1-\frac{1}{2 \tau_{T}}\right) \partial_{t}(\mathbf{u} T) .
$$

Taking this correction into account makes the scheme secondorder accurate under both convective and diffusive scaling 30.

Comparing the recovered PDE to the energy conservation equation, i.e. Eq. 2 one can observe that the advection operator in the LB advection-diffusion model becomes consistent only in the limit of a strictly incompressible flow:

$$
\nabla \cdot \mathbf{u} T=\mathbf{u} \cdot \nabla T+T \nabla \cdot \mathbf{u},
$$

The classical LB flow solver not being strictly incompressible, $\nabla \cdot \mathbf{u} \neq 0$ 31. For flows involving thermo-compression, such as those present in low Mach combustion applications (corresponding to the Low Mach Number Approximation - LMNA), both the time evolution and advection operators lack a density term.

Apart from the above-mentioned error, the classical LB approach also admits another source of error in the diffusion term; The diffusion term recovered by this model is only correct for incompressible flows with constant specific heat capacity. If we were to assume that the heat capacity and density are not constant (therefore replace $c_{p, 0}$ and $\rho_{0}$ with $c_{p}$ and $\rho$ ) the diffusion term (as recovered by the classical approach) would be:

$$
\nabla \cdot\left(\frac{\lambda}{\rho c_{p}} \nabla T\right)=\frac{1}{\rho c_{p}} \nabla \cdot(\lambda \nabla T)+(\lambda \nabla T) \cdot\left(\nabla \frac{1}{\rho c_{p}}\right) .
$$
heat capacity distribution over the simulation domain, i.e. for $c_{p}=c_{p, 0}=$ const.

As mentioned earlier, this results in inconsistent diffusive fluxes at solid-fluid interfaces in conjugate heat transfer cases where the solid and fluid have different specific heat capacities or densities. A modified model is proposed in the next section to overcome these issues and make the LB advection-diffusion scheme suitable for heat transfer in heterogeneous media.

\section{3. Modified heat transport LB model}

\subsection{Brief overview of $L B$ flow solver}

Although used in this work without any modification, the classical single relaxation lattice Boltzmann model formulation used for the flow simulation will be briefly summarized in this subsection. The classical LB solver is usually employed to solve the Navier-Stokes equations in the limit of incompressible flows. The discrete time-evolution operator of the populations, $f_{\alpha}(\mathbf{x}, t)$, is given as:

$$
\begin{aligned}
f_{\alpha}\left(\mathbf{x}+\mathbf{c}_{\alpha} \delta_{t}, t+\delta_{t}\right)- & f_{\alpha}(\mathbf{x}, t)= \\
& \frac{1}{\tau_{F}}\left(f_{\alpha}^{(e q)}(\mathbf{x}, t)-f_{\alpha}(\mathbf{x}, t)\right),
\end{aligned}
$$

where $f_{\alpha}^{(e q)}(\mathbf{x}, t)$ is the flow solver EDF and $\tau_{F}$ is the corresponding relaxation coefficient. The equilibrium distribution function is usually taken as:

$$
\begin{aligned}
f_{\alpha}^{(e q)}(\mathbf{x}, t)=w_{\alpha} \rho(\mathbf{x}, t) & \left(1+\frac{\mathbf{c}_{\alpha} \cdot \mathbf{u}(\mathbf{x}, t)}{c_{s}^{2}}\right. \\
& \left.+\frac{\left(\mathbf{c}_{\alpha} \cdot \mathbf{u}(\mathbf{x}, t)\right)^{2}}{2 c_{s}^{4}}-\frac{\mathbf{u}(\mathbf{x}, t)^{2}}{2 c_{s}^{2}}\right),
\end{aligned}
$$

while the relaxation coefficient is computed as:

$$
\tau_{F}=\frac{1}{\delta_{t} c_{s}^{2}} \nu+\frac{1}{2}
$$

where $\nu$ is the fluid kinematic viscosity. The density and fluid velocity can be obtained as moments of the distribution function:

$$
\begin{gathered}
\sum_{\alpha} f_{\alpha}(\mathbf{x}, t)=\rho(\mathbf{x}, t) \\
\frac{1}{\rho} \sum_{\alpha} \mathbf{c}_{\alpha} f_{\alpha}(\mathbf{x}, t)=\mathbf{u}(\mathbf{x}, t)
\end{gathered}
$$

Further details on the employed LB solver can be found in 32 .

\subsection{Proposed solver for energy field}

In order for the model to be able to deal with changes in specific heat capacity an automata recovering a conservative form of the total sensible energy transport equation is proposed. The time evolution operator is similar to the classical collision-streaming equation:

$$
\begin{aligned}
& h_{\alpha}\left(\mathbf{x}+\mathbf{c}_{\alpha} \delta_{t}, t+\delta_{t}\right)-h_{\alpha}(\mathbf{x}, t)= \\
& \frac{1}{\tau_{H}}\left(h_{\alpha}^{(e q)}(\mathbf{x}, t)-h_{\alpha}(\mathbf{x}, t)\right)+w_{\alpha} \dot{\omega}_{T}(\mathbf{x}, t)+w_{\alpha} \mathbf{c}_{\alpha} \cdot \mathbf{F},
\end{aligned}
$$

where $\tau_{H}$ is the relaxation coefficient given later on in this subsection. The conserved zeroth-order moment of the new distribution function is defined to be:

$$
\sum_{\alpha} h_{\alpha}=H_{t}
$$

where $H_{t}$, the conserved variable, is the total energy minus the enthalpy of formation per unit volume, defined as:

$$
H_{t}=\rho \epsilon+\frac{\rho \mathbf{u}^{2}}{2}+p-\rho \Delta h_{f}^{0}\left(T_{0}\right),
$$

where $\epsilon$ is the internal energy per unit mass and $\Delta h_{f}^{0}$ is the mixture enthalpy of formation per unit mass at a reference temperature $T_{0}$ and $p$ is the pressure. For the rest of this work pressure contribution to total enthalpy will be neglected, which is acceptable for most practical applications in the limit of incompressible flows (since the classical LB solver employed here is a weakly compressible 
solver valid only for low Mach numbers). Based on this, and assuming first for the sake of clarity that the specific heat capacity is not a function of temperature, the local temperature can be obtained as a moment of the distribution function:

$$
T=\left(\frac{1}{\rho} \sum_{\alpha} h_{\alpha}-\frac{\mathbf{u}^{2}}{2}\right) / c_{p}
$$

For cases where the specific heat capacity is a function of the local temperature, the temperature can be recovered through an iterative root-finding algorithm, such as the Newton-Raphson method. Assuming that the total sensible enthalpy $H_{t}$ at a given point in space, $\mathbf{x}$, and time, $t$, is known, one can find the local temperature by finding the root of the following equation:

$$
f(T)=H_{t}(\mathbf{x}, t)-\frac{\rho(\mathbf{x}, t) \mathbf{u}^{2}(\mathbf{x}, t)}{2}-\rho(\mathbf{x}, t) \int_{T_{0}}^{T} c_{p}(T) d T .
$$

Starting from an initial guess for the temperature - the local temperature at the previous time-step being a natural choice, one can get successively better approximations at the $n+1^{\text {th }}$ iteration using the following equation:

$$
T^{n+1}(\mathbf{x}, t)=T^{n}(\mathbf{x}, t)+\frac{f\left(T^{n}(\mathbf{x}, t)\right)}{c_{p}\left(\rho(\mathbf{x}, t) T^{n}(\mathbf{x}, t)\right)} .
$$

The root-finding algorithm is used in all test-cases presented in this work. It is worth mentioning that on average 2 iterations are sufficient for the algorithm to converge at each point. To recover the correct physics at the macro- ${ }^{45}$ scopic scale the EDF is defined as:

$$
\begin{array}{r}
h_{\alpha}^{(e q)}(\mathbf{x}, t)=w_{\alpha} H_{t}(\mathbf{x}, t)\left(1+\frac{1}{c_{s}^{2}} \mathbf{c}_{\alpha} \cdot \mathbf{u}(\mathbf{x}, t)\right) \\
+\eta_{\alpha}\left(\gamma T(\mathbf{x}, t)-H_{t}(\mathbf{x}, t)\right),
\end{array}
$$

where $\gamma$ is a free parameter fixed by the user at the beginning of the simulation and uniform in space, with unit $\mathrm{kg} / \mathrm{K} . \mathrm{m} . \mathrm{s}^{2}$. The parameter $\gamma$ is introduced here in the model for the sake of stability, while $\eta_{\alpha}$ are weights subject to the following restrictions [33]:

$$
\begin{gathered}
\sum_{\alpha} \eta_{\alpha}=0, \\
\sum_{\alpha} \mathbf{c}_{\alpha} \eta_{\alpha}=0, \\
\sum_{\alpha} \mathbf{c}_{\alpha} \otimes \mathbf{c}_{\alpha} \eta_{\alpha}=c_{s}^{2} \mathbf{I} .
\end{gathered}
$$

In order to satisfy the above-mentioned restrictions, for the rest of this work the second weight coefficients are defined as:

$$
\begin{gathered}
\eta_{0}=-\sum_{\alpha \neq 0} w_{\alpha}, \\
\forall \alpha \neq 0, \eta_{\alpha}=w_{\alpha} .
\end{gathered}
$$

Using Eqs. 19 through 30 and the multi-scale ChapmanEnskog analysis method, it can be shown that up to second order the following macroscopic equation is recovered by the proposed scheme (a detailed analysis is given in Appendix A):

$$
\partial_{t} H_{t}+\nabla \cdot\left(H_{t} \mathbf{u}\right)-\nabla \cdot \lambda \nabla T-\dot{\omega}_{T}=0
$$

The production term, $\dot{\omega}_{T}$ is defined as:

$$
\dot{\omega}_{T}=\rho \sum_{k} \dot{\omega}_{k} M_{k} \Delta h_{f, k}^{0}\left(T_{0}\right)
$$

where $\dot{\omega}_{k}$ is the $\mathrm{k}^{\text {th }}$ species mole production rate and $M_{k}$ is the molar mass. In order to recover the correct diffusion term, the relaxation coefficient must be set to:

$$
\tau_{H}=\frac{1}{\delta_{t} \gamma c_{s}^{2}} \lambda+\frac{1}{2}
$$

Just as for the classical AD-LB approach, to take out the first-order error term (in space and time) and get Eq. 31 up to second order, the force term has to be set to:

$$
\mathbf{F}=\frac{1}{c_{s}^{2}}\left(1-\frac{1}{2 \tau_{H}}\right) \partial_{t}\left(H_{t} \mathbf{u}\right) .
$$

It is also worth mentioning that in the context of the present study, the time derivative in the force term is computed using a first-order finite-difference approximation:

$$
\partial_{t}\left(H_{t} \mathbf{u}\right)=\frac{H_{t}(t) \mathbf{u}(t)-H_{t}\left(t-\delta_{t}\right) \mathbf{u}\left(t-\delta_{t}\right)}{\delta_{t}}+\mathcal{O}\left(\delta_{t}^{2}\right)
$$

\subsection{Algorithmics and coupling}

In the context of the model presented in this work, the flow and energy solvers operate in parallel and are explicitly coupled. At the beginning of each time-step, using data from the previous time-step, first the local thermodynamics and transport properties are computed. Then, the populations at the new time-step for each solver (flow and energy) are computed. Finally, the different moments of the distribution functions are extracted. The code structure is illustrated in Fig. 1 .

\section{Further details about the proposed model}

\subsection{Simplified forms of the scheme}

For flows where the local specific heat capacity is only function of temperature, density variations are negligible and viscous heat dissipation is of minor importance, the proposed scheme can be simplified by taking the following definition for the EDF:

$$
h_{\alpha}^{(e q)}(\mathbf{x}, t)=w_{\alpha} h_{s}(\mathbf{x}, t)\left(1+\frac{1}{c_{s}^{2}} \mathbf{c}_{\alpha} \cdot \mathbf{u}(\mathbf{x}, t)\right),
$$

where the sensible enthalpy per unit mass, $h_{s}$, is now the conserved variable. Through this EDF, the following PDE can be recovered:

$$
\partial_{t} h_{s}+\nabla \cdot \mathbf{u} h_{s}-\nabla \delta_{t} c_{s}^{2} \frac{2 \tau_{h s}-1}{2} \nabla h_{s}-\frac{\dot{\omega}_{T}}{\rho_{0}}=0
$$




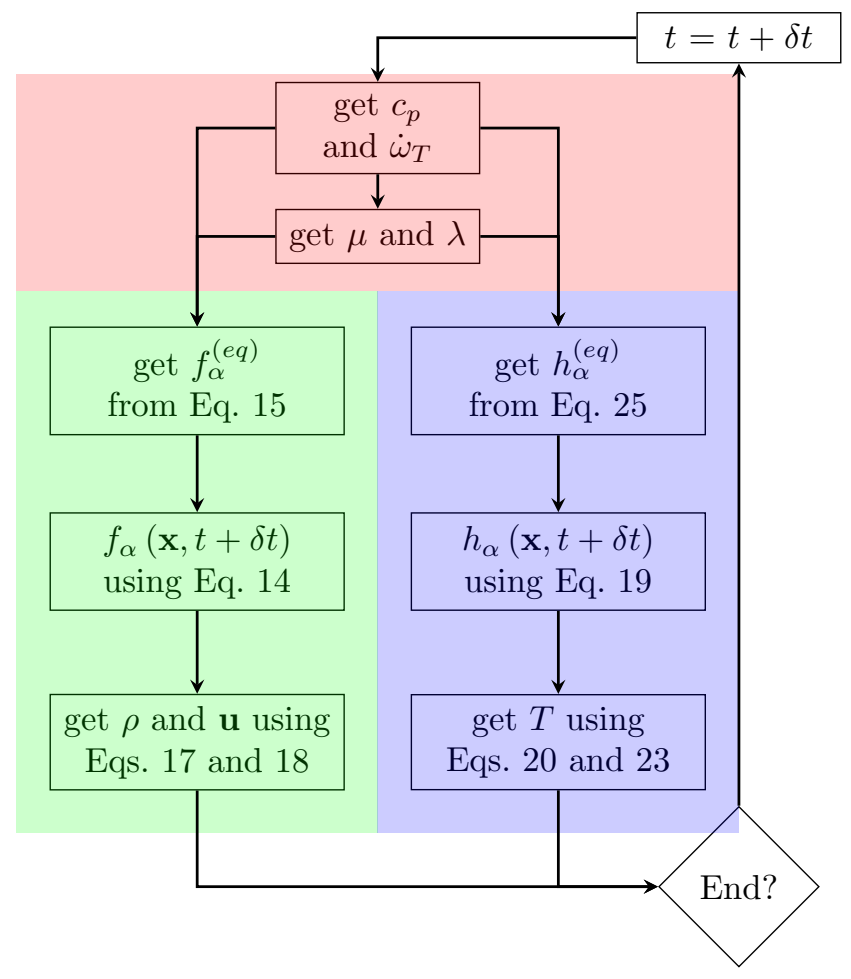

Figure 1: Overall structure of the proposed simulation scheme

Using:

$$
\nabla h_{s}=\underbrace{\frac{\partial h_{s}}{\partial T}}_{c_{p}} \nabla T
$$

and:

$$
\tau_{h s}=\frac{\lambda}{\rho_{0} c_{p} c_{s}^{2} \delta_{t}}+\frac{1}{2}
$$

one gets the following PDE:

$$
c_{p} \partial_{t} T+c_{p} \nabla \cdot \mathbf{u} T-\nabla \frac{\lambda}{\rho_{0}} \nabla T-\frac{\dot{\omega}_{T}}{\rho_{0}}=0 .
$$

In cases where sensible enthalpy is not only a function of local temperature, for example for conjugate heat transfer where $h_{s}=h_{s}(\mathbf{x}, T)$, Eq. 38 does not hold anymore. For flows involving multiple species, $h_{s}=h_{s}\left(Y_{0}, \ldots, Y_{k}, T\right)$, Eq. 38 takes the following form:

$$
\nabla h_{s}=\frac{\partial h_{s}}{\partial T} \nabla T+\sum_{k=1}^{N_{s p}} \frac{\partial h_{s}}{\partial Y_{k}} \nabla Y_{k}
$$

which in turn would lead to the need for an additional, complex correction term.

For cases involving viscous dissipation, choosing sensible enthalpy as the conserved variable, ignoring pressure work and assuming a single-component flow, would lead to the following PDE:

$$
\partial_{t} \rho h_{s}+\nabla \cdot \rho h_{s} \mathbf{u}-\nabla \lambda \nabla T-\phi=0 .
$$

where viscous dissipation, $\phi$, appears in a non-conservative form:

$$
\phi=(\mathbf{S} \cdot \nabla) \cdot \mathbf{u}
$$

where $\mathbf{S}$ is the viscous stress tensor. Deriving such terms in LB is usually complicated and leads to additional nonlocal operations. Hence, for cases involving viscous dissipation total energy is the better choice. The current study will focus on the energy balance equation ignoring viscous dissipation. The addition of viscous dissipation and derivation of the corresponding terms in the context of this model will be the subject of our future work.

\subsection{Stability, errors and free parameter}

The free parameter $\gamma$ in the EDF of Eq. 25 is subject to certain restrictions to avoid negative distribution functions and large error terms. In order to have a stable simulationfree from Gibbs oscillations, the relaxation coefficient must not come too close to $1 / 2$. Simultaneously, positivity of the EDF must be guaranteed; for an advection-diffusion LB model, small relaxation coefficients along with negative EDFs can lead to instabilities 34. Studying the EDFs one can see that for resting particles, the following positivity condition is obtained:

$$
H_{t} \underbrace{\left(w_{0}+\sum_{\alpha \neq 0} w_{\alpha}\right)}_{=1} \geq \gamma\left(\sum_{\alpha \neq 0} w_{\alpha}\right) T,
$$

while for moving particle distributions - non-zero directions, the positivity condition changes to:

$$
\left(\frac{\gamma T}{H_{t}}\right) c_{s}^{2} \geq\|\mathbf{u}\|
$$

The total sensible enthalpy $H_{t}$ and temperature $T$ appearing in these equations should be those resulting in the smallest value for the ratio $T / H_{t}$.

Furthermore, one must keep in mind that large relaxation coefficients $-\tau_{H} \gg 1$ - can have negative and noticeable impact on higher-order errors. Those can be expressed -assuming constant $\tau_{H^{-}}$as:

$$
\Delta=\sum_{i=3}^{\infty} \epsilon^{i} R_{(i)}\left(\tau_{H}\right)\left(\partial_{t}+\nabla \cdot \mathbf{c}_{\alpha}\right)^{i} \mathbf{c}_{\alpha} h_{\alpha}^{(e q)}
$$

where $R_{(i)}\left(\tau_{H}\right)$ are the Chapman polynomials [35]. At $i=3-5$ for example one has [36]:

$$
\begin{gathered}
R_{(3)}\left(\tau_{H}\right)=\tau_{H}^{2}-\tau_{H}+\frac{1}{6}, \\
R_{(4)}\left(\tau_{H}\right)=-\tau_{H}^{3}+\frac{3}{2} \tau_{H}^{2}-\frac{7}{12} \tau_{H}+\frac{1}{24}, \\
R_{(5)}\left(\tau_{H}\right)=\tau_{H}^{4}-2 \tau_{H}^{3}+\frac{5}{4} \tau_{H}^{2}-\frac{1}{4} \tau_{H}+\frac{1}{120},
\end{gathered}
$$

which obviously will grow much faster than the diffusion term for large values of $\tau_{H}$. 
As such, the free parameter $\gamma$ must be chosen as an opti70 mum value with regard to all of the aforementioned issues. Although not strictly bound by the previously mentioned conditions, they can be seen as sufficient conditions for a stable simulation. The effects of the choice of $\gamma$ are better illustrated through test-cases in the upcoming sections.

75 In the next section we will study the performances of the code in the face of variations in density, specific heat capacities and thermal conductivity.

\section{Numerical validations and results}

In this section, the proposed scheme is confronted to a 80 variety of situations, to showcase its ability to model complex thermal flows. First, through cases involving spacevarying thermal conductivity -both continuous and subject to discontinuity, the ability of the model to deal with variable thermal conductivity is established. Then, un-

85 steady heat conduction in a 3-layer medium with different thermal conductivities and specific heat capacities is modeled to show that, contrary to the classical advectiondiffusion scheme, the proposed approach correctly takes into account variations in specific heat capacity. This 90 test-case is also used to illustrate the effect of the free tunable parameter on stability and error. Finally, to establish the robustness of the model three different heat transfer problems of increasing complexity involving conduction and convection by fluid flow are considered: (1)

95 heated lid-driven cavity with temperature-dependent thermal conductivity, specific heat capacity and viscosity, (2) 2 -D rectangular channel with mounted obstacles on upper and lower walls subject to heat flux at their bases, and (3) turbulent flow over a multi-layered wall-mounted cube.

\subsection{Steady-state conduction in medium with variable dif-} fusion coefficient

In order to show the ability of the scheme to properly model heat diffusion when subject to variations in thermal conductivity, two test-cases are studied here.

\subsubsection{Two-layer media with discontinuous thermal con- ductivity}

First a two-layer medium is considered. The simulation domain is composed of two solid regions, with the same heat capacity and density, but different thermal diffusion coefficients. The upper and lower boundaries are set to constant temperatures, $T_{h}$ and $T_{c}$ respectively, while other boundaries are treated as zero-flux. The case is defined through the following equations:

$$
\begin{gathered}
\partial_{t} T-\partial_{y} \lambda \partial_{y} T=0, \\
\lambda=\left\{\begin{array}{l}
\lambda=\lambda_{1}, 0 \leq y<L_{1}, \\
\lambda=\lambda_{2}, L_{1} \leq y \leq L .
\end{array}\right. \\
T(y=0)=T_{c},
\end{gathered}
$$

$$
T(y=L)=T_{h}
$$

where $L$ is the size of the domain, $L_{1}$ is the length of the first zone and $\lambda_{1}$ and $\lambda_{2}$ are respectively the first and second zone thermal conductivities. The test-case configuration is illustrated in Fig. 2 .

The analytical steady-state solution for this configuration

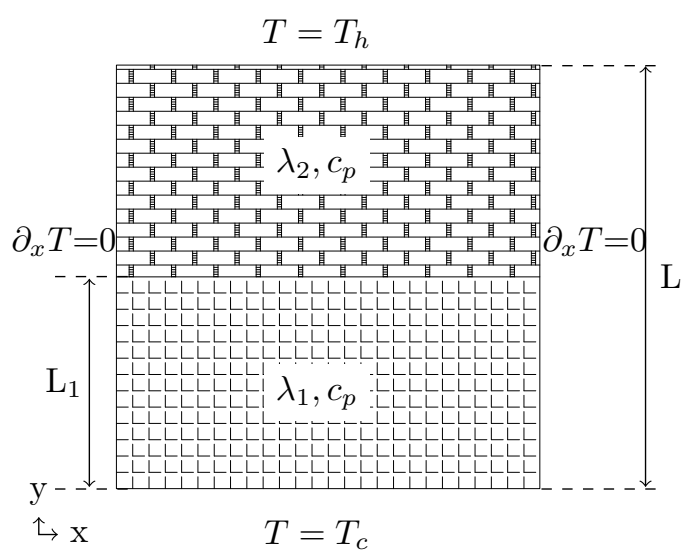

Figure 2: Two-block solid case configuration

can be found to be:

$T(y)=\left\{\begin{array}{l}\frac{\left(T_{h}-T_{c}\right) y}{\lambda_{1}\left(\frac{L_{1}}{\lambda_{1}}+\frac{L-L_{1}}{\lambda_{2}}\right)}+T_{c}, y<L_{1} \\ \frac{\left(T_{h}-T_{c}\right) y}{\lambda_{2}\left(\frac{L_{1}}{\lambda_{1}}+\frac{L-L_{1}}{\lambda_{2}}\right)}+T_{h}-\frac{\left(T_{h}-T_{c}\right) L}{\lambda_{2}\left(\frac{L_{1}}{\lambda_{1}}+\frac{L-L_{1}}{\lambda_{2}}\right)}, L_{1} \leq y \leq L\end{array}\right.$

The domain length, $L$, was set to $1 \mathrm{~m}$ while the length of the first block, $L_{1}$, was taken as $0.5 \mathrm{~m}$. The upper and lower temperatures, $T_{h}$ and $T_{c}$ were respectively set to $1000 \mathrm{~K}$ and $300 \mathrm{~K}$. The thermal conductivities, $\lambda_{1}$ and $\lambda_{2}$ were taken respectively as $1 \times 10^{-3}$ and $2 \times 10^{-3}$ $\mathrm{W} / \mathrm{m} . \mathrm{K}$, while $\delta_{t}$ was set to $0.01 \mathrm{~s}, \delta_{x}$ to $0.01 \mathrm{~m}$, and $\gamma$ to 1 . This choice of parameters results in a relaxation parameter of $\tau_{H}=0.8-1.1$. For these values the simulations are stable, while small values of the relaxation constant usually result in Gibbs oscillations, and large values lead to divergence (just like classical solvers for advectiondiffusion equations). Furthermore, the CFL-like condition expressed by Eq. 45 does not need to be considered for this case, as there is no convection. The simulation was initialized with a uniform temperature distribution, $T=T_{c}$, and carried out on a D2Q5 stencil. Fixed temperature boundary conditions were imposed using the anti-bounce-back approach 37] while zero-flux boundaries were applied to the missing populations following [37]:

$$
g_{\alpha}\left(\mathbf{x}, t+\delta_{t}\right)=g_{\alpha}\left(\mathbf{x}-\mathbf{n} \delta_{t}, t+\delta_{t}\right),
$$

where $\mathbf{n}$ is the outward-pointing unit vector perpendicular to the zero-gradient boundary. The results obtained from simulation using the proposed LB model are compared to the analytical solution in Fig. 3. To better quantify the 


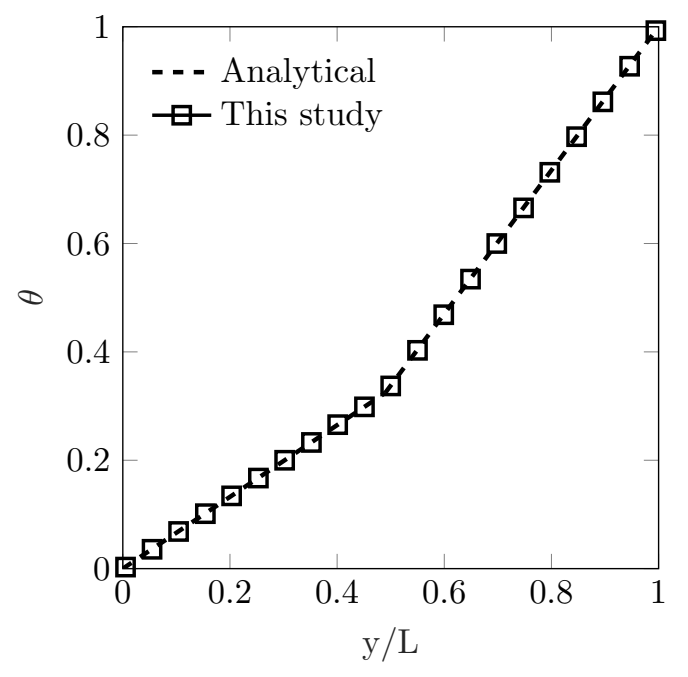

Figure 3: Profile of steady-state non-dimensional temperature, $\theta=$ $\frac{T-T_{c}}{T_{h}-T_{c}}$, along the vertical centerline for the two-block media case

accuracy of the results, the normalized $L_{2}$ and $L_{\infty}$ norms of the temperature field were computed as:

$$
L_{2}=\frac{\sqrt{\frac{1}{N} \sum_{i}\left(T_{i}-T_{i, r e f}\right)^{2}}}{\sqrt{\frac{1}{N} \sum_{i} T_{i, r e f}^{2}}},
$$

and :

$$
L_{\infty}=\frac{\max \left|T_{i}-T_{i, r e f}\right|}{\sqrt{\frac{1}{N} \sum_{i} T_{i, r e f}^{2}}} .
$$

This test-case yielded $1.674 \times 10^{-5}$ and $2.438 \times 10^{-4}$ for $_{115}$ the $L_{2}$ and $L_{\infty}$ norms, respectively.

\subsubsection{Couette flow with variable thermal conductivity}

The second configuration considered in this section is a 2-D thermal Couette flow with a temperature-dependent thermal conductivity. Just as before upper and lower boundary temperatures are set to $T_{h}$ and $T_{c}$ respectively while other boundaries are periodic. The flow field is subject to a constant velocity at the upper boundary. The steadystate configuration of the thermal field is defined through the following system of equations:

$$
\begin{gathered}
\partial_{y} \lambda \partial_{y} T=0, \\
\lambda=\frac{\lambda_{0} T}{T_{0}}, \\
T(y=0)=T_{c}, \\
T(y=L)=T_{h}
\end{gathered}
$$

where $\lambda_{0}$ is the thermal conductivity at a reference temperature $T_{0}$. The simulation was carried out on a D2Q9 stencil for the flow field and D2Q5 stencil for the energy

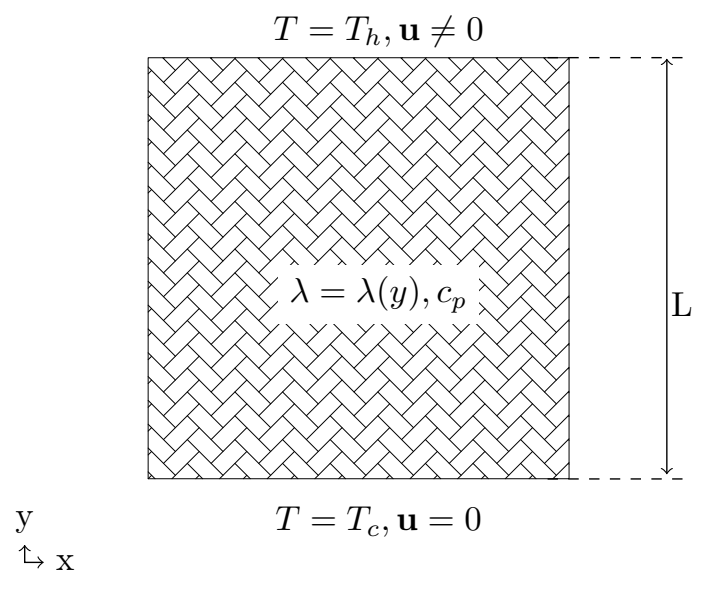

Figure 4: Thermal Couette flow case configuration

field. The overall configuration is given in Fig. 4

Given the lack of convective heat flux in the x-direction, the steady-state solution reduces to that of a solid media with variable thermal conductivity:

$$
T(y)=\sqrt{\left(\frac{y}{L} T_{h}^{2}+\frac{(L-y)}{L} T_{c}^{2}\right)} .
$$

The domain length, $L$, is set to $1 \mathrm{~m}$. The upper and lower temperatures, $T_{h}$ and $T_{c}$ were respectively set to $1000 \mathrm{~K}$ and $300 \mathrm{~K}$. The thermal conductivity $\lambda_{0}$ at temperature $T_{0}=300 \mathrm{~K}$, was set to $1 \times 10^{-3} \mathrm{~W} / \mathrm{m} . \mathrm{K}$, while $\delta_{t}$ was fixed at $0.01 \mathrm{~s}, \delta_{x}$ at $0.01 \mathrm{~m}$ and $\gamma$ at 1 , which resulted in $\tau_{H}=0.8-1.5$. Stationary and moving-wall boundary conditions were modeled using the half-way bounceback and modified half-way bounce back methods respectively. Figure 5 compares the temperature profiles along

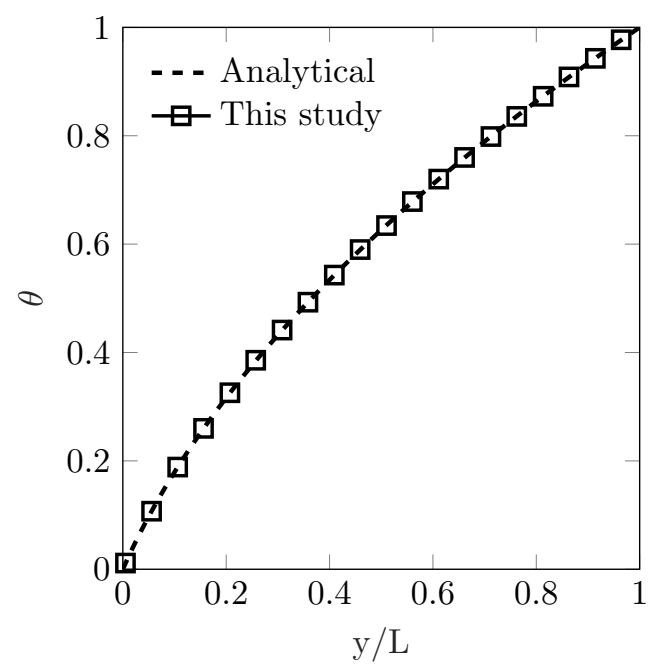

Figure 5: Steady-state non-dimensional temperature, $\theta=\frac{T-T_{c}}{T_{h}-T_{c}}$, profile along the vertical centerline for the thermal Couette flow

the vertical centerline to the analytical solution of Eq. 62 . 
As shown in this figure the model is able to correctly capture the effects of variable thermal conductivity with fixed density and heat capacity. Furthermore, the obtained $L_{2}$ and $L_{\infty}$ norms are respectively $2.24 \times 10^{-4}$ and $4.90 \times 10^{-3}$. It is also worth mentioning that noticeable erare found only near the boundary conditions, caused by the relaxation-coefficient dependence of the position of the wall when bounce-back schemes are used. The next subsection will focus on the unsteady performance of the model when subject to both changes in thermal conduc-

\subsection{Transient conduction in three-layer media with differ- ent thermal conductivities and specific heat capacities}

One of the main advantages of the model presented in this work is that it effectively allows for variable heat capacities in the simulation. To showcase the ability of the model to reproduce correct results even when subject to discontinuities in both heat conductivity and specific heat capacity, the case of transient heat conduction in a threeblock solid domain is considered here 38. The temperatures on the upper and lower boundaries of the domain are fixed at $T_{h}$ and $T_{c}$. The domain is composed of three different regions with different specific heat capacities and thermal conductivities. The corresponding configuration is illustrated in Fig. 6. Initially, the temperature is set to $\theta=0$ everywhere in the domain, where $\theta=\frac{T-T_{c}}{T_{h}-T_{c}}$. The theoretical temperature profiles at different times are

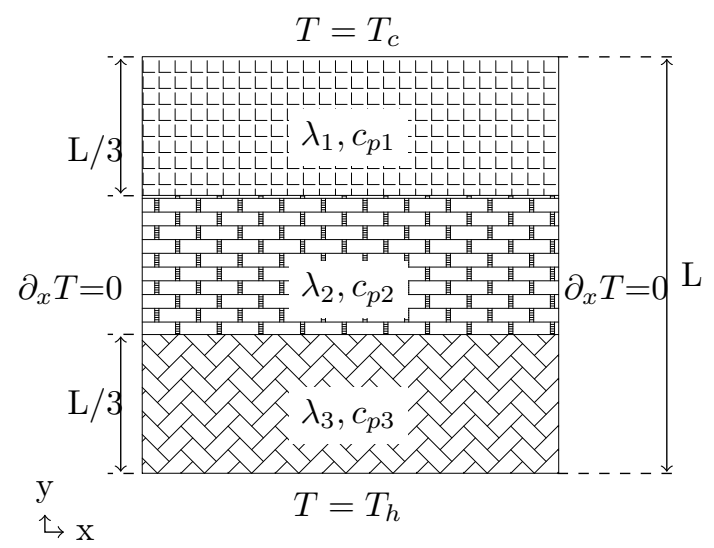

Figure 6: Three-block solid media test-case configuration

reported in [38]. The different physical parameters used ${ }^{135}$ in the context of the present study are listed in Table 1. The upper and lower boundaries temperatures are set to

\begin{tabular}{||c||c|c|c||}
\hline Species & $\lambda[\mathrm{W} / \mathrm{m} . \mathrm{K}]$ & $c_{p}[\mathrm{~J} / \mathrm{kg} . \mathrm{K}]$ & $\mathrm{L}[\mathrm{m}]$ \\
\hline \hline Zone 1 & 1. & 1. & 1. \\
\hline Zone 2 & 0.1 & 0.033 & 1. \\
\hline Zone 3 & 1. & 1. & 1. \\
\hline
\end{tabular}

Table 1: Simulation parameters for unsteady three-block media testcase 38

$T_{h}=1000 \mathrm{~K}$ and $T_{c}=300 \mathrm{~K}$ while $\delta_{x}$ and $\delta_{t}$ are respectively set to $3.33 \times 10^{-2} \mathrm{~m}$ and $1 \times 10^{-5} \mathrm{~s}$. In order for the EDF to remain positive and guarantee stability the parameter $\gamma$ was set to 0.05 . Boundary conditions on the temperature field were imposed using the schemes detailed in the previous subsection. The temperature profiles at different times, namely $0.1,0.5,1$ and $2 \mathrm{~s}$ are compared to their reference counterparts in Fig. 7. As mentioned in

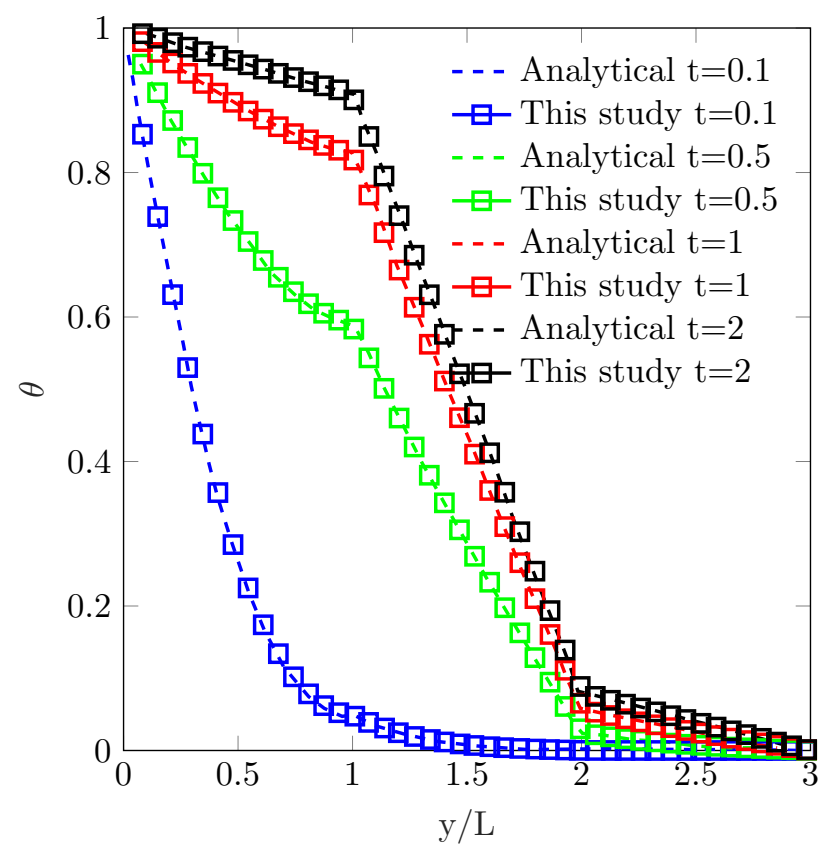

Figure 7: Temperature profile at different times for the three-block solid media case, theoretical solutions are taken from 38

24, the classical advection-diffusion LB model can not reproduce the correct results for this case. At the interfaces, the classical model recovers the following flux:

$$
\mathbf{q}=-\frac{\lambda}{\rho c_{p}} \nabla T
$$

instead of the correct Fourier flux:

$$
\mathbf{q}=-\lambda \nabla T
$$

The proposed model, on the other hand, recovers the correct flux and as such can deal with variations in both specific heat capacity and thermal conductivity. As seen in Fig. 7 the obtained profiles are in excellent agreement with their analytical counterparts. Test-cases presented up to this point have established that the proposed scheme correctly models thermal systems subject to variable thermal conductivity and specific heat capacity, even with a convective field perpendicular to the diffusion flux vector.

In the previous sections, the choice of the free parameter $\gamma$ was shown to be important regarding stability and leading-order error terms. To better illustrate this effect the present test-case was run for different values of $\gamma$ without changing any of the remaining parameters. In this 
context the value of $\gamma$ was changed to span a rather large interval, between 1 and $5 \times 10^{-3}$. The results of these

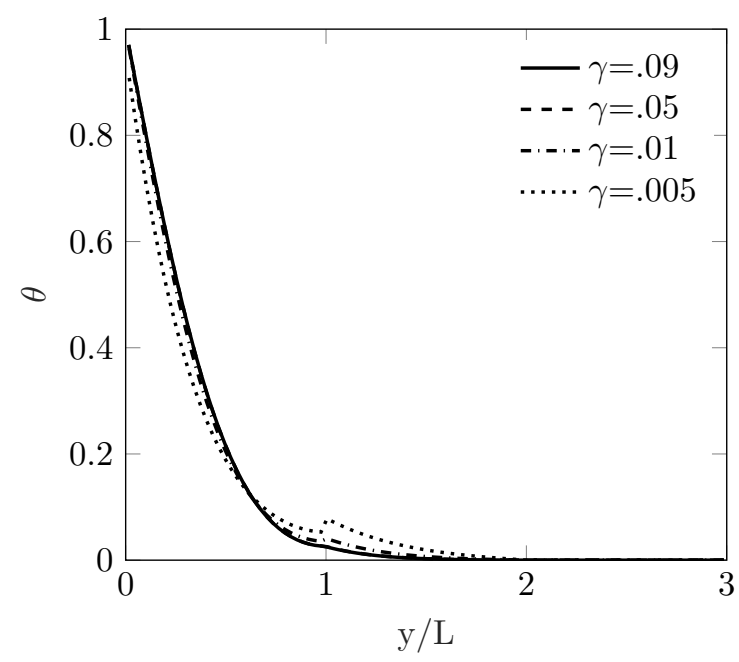

Figure 8: Temperature profile at at $t=0.1 \mathrm{~s}$ for different values of $\gamma$

simulations at $t=0.1 \mathrm{~s}$, are shown in Fig. 8. Only results

from simulations with $\gamma \leq 0.09$ are displayed, as larger values led to unstable simulations (The simulations did not reach $t=0.1 \mathrm{~s})$. To better explain the unstable simulations and establish a link with Eq. 44, additional data and ${ }^{180}$ parameters from these simulations are given in Table 2 .

\begin{tabular}{||c|c|c|c||}
\hline$\gamma$ & $\tau_{H}$ & $\frac{\gamma \sum_{\alpha \neq 0} w_{\alpha}}{c_{p}}$ & $L_{2}$ \\
\hline \hline 0.09 & $0.53-0.8$ & $0.06-1.82$ & $2.87 \times 10^{-5}$ \\
\hline 0.05 & $0.55-1.04$ & $0.03-1.01$ & $9.66 \times 10^{-4}$ \\
\hline 0.01 & $0.77-3.21$ & $0.007-0.20$ & $3.49 \times 10^{-2}$ \\
\hline 0.005 & $1.04-5.91$ & $0.003-0.10$ & $1.43 \times 10^{-1}$ \\
\hline
\end{tabular}

Table 2: Simulation parameters for transient heat conduction in $\mathrm{n}_{190}$ three-layer media test-case with varying $\gamma$

In this table, the range of the relaxation coefficient in the domain along with $\frac{\gamma \sum_{\alpha \neq 0} w_{\alpha}}{c_{p}}$ and the errors are shown. It is observed that above $\gamma=0.05$ the stability condi-195 tion of Eq. 44 is not satisfied, while below 0.01 the maximum relaxation coefficient in the domain becomes rather large. This in turn explains the onset of instability at $\gamma=0.09$ and the very pronounced error at $y / l=1$ for $\gamma=0.005$. The conditions provided for the free parameter ${ }^{200}$ $\gamma$ can therefore be seen as useful to ensure stability and minimize the error coming from higher-order terms. Now that the role of the free parameter has also been clarified, the next subsections will focus on more general cases.

\subsection{Heated lid-driven cavity}

To better establish the ability of the proposed solver to model realistic configurations, the case of a 2-D heated walls, the temperature is fixed at $T_{c}$. The upper boundary on the other hand is a moving wall, with velocity $u_{0}$. The upper-wall temperature is fixed at $T_{h}$. Given the complex-

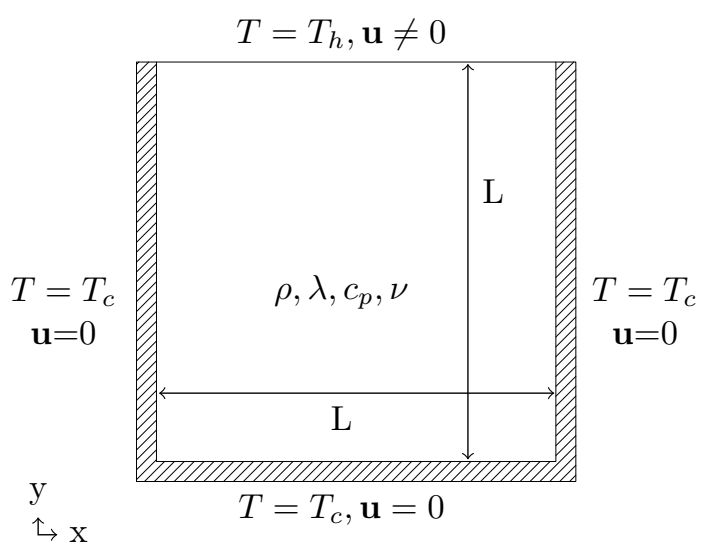

Figure 9: Heated lid-driven cavity configuration

ity of the case, especially when subject to variable transport coefficients, no analytical solution can be proposed. Instead, to validate the result, temperature and velocity profiles are compared here to a corresponding simulation using the well-established tool ANSYS-Fluent. Two different configurations are considered here: (a) a configuration 185 where all parameters, i.e. $\nu, \lambda$ and $c_{p}$ are fixed, and (b) a case where all parameters are temperature-dependent. For the first configuration, the Reynolds is fixed at 400 and the Prandtl number at 1 . The upper wall temperature is set to $1500 \mathrm{~K}$ while all other boundaries are fixed at $T_{c}=300$ $\mathrm{K}$. The simulation is performed on a $200 \times 200$ uniform grid for both LB and Fluent. As for previous cases, D2Q9 and D2Q5 stencils are used respectively for the flow and temperature fields. To follow the test-case presented in [28, the wall velocity is set to $0.1 \mathrm{~m} / \mathrm{s}$, dynamic viscosity at the reference temperature, $T_{0}=T_{c}$ is set to $\mu_{0}=0.05$ $\mathrm{kg} / \mathrm{m} . \mathrm{s}$, density is set to $1 \mathrm{~kg} / \mathrm{m}^{3}, \lambda_{0}$ to $0.125 \mathrm{~W} / \mathrm{m} . \mathrm{K}$ and $\delta_{x}$ and $\delta_{t}$ are both set to 1 (respectively in meters and seconds) while $\gamma$ is set to 10 . To better observe the changes induced by variable flow properties both flow and temperature fields are compared in Fig. 10. Due to the temperature-dependence of the viscosity, as observed in this figure, the flow structure is affected. For example the centers of the vortices on the lower left and right corners have been displaced. 

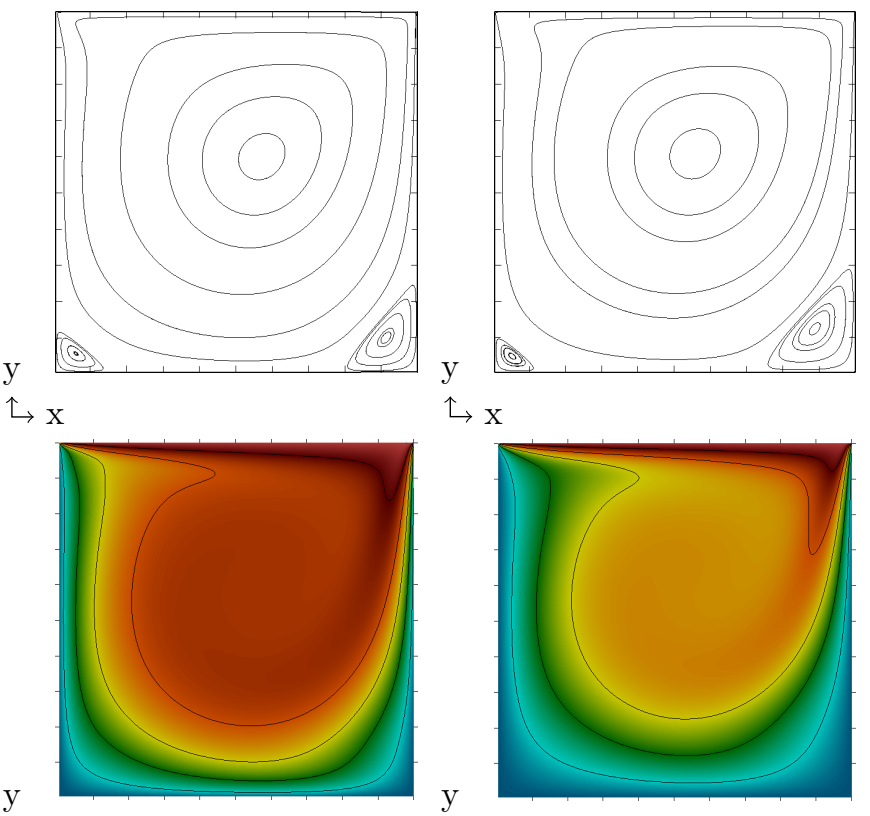

$\uparrow \mathrm{x}$

$\uparrow \mathrm{x}$

Figure 10: Heated lid-driven cavity flow structure. Top to bottom: stream-lines and temperature distribution. Left to right: constant and variable fluid properties

The velocity and temperature profiles along the vertical and horizontal centerlines are compared to their reference counter-parts in Figs. 11 and 12 . As expected, the LB solver recovers both the correct velocity and temperature ${ }_{225}$ fields

Next, the same configuration is subjected to variable transport and thermodynamic properties. These parameters are defined through the following equations, similar to those used in [28]:

$$
\begin{gathered}
\mu(T)=\frac{\mu_{0} T}{2 T_{0}} \\
\lambda(T)=\frac{\lambda_{0} T}{T_{0}} \\
c_{p}(T)=0.5632 \frac{T-T_{0}}{T_{0}}+c_{p, 0}
\end{gathered}
$$

where $c_{p 0}$ is set to $2.5 \mathrm{~J} / \mathrm{kg} . \mathrm{K}$ following [28]. As for the constant-properties case, the simulation was performed on a $200 \times 200$ grid. The results obtained from the LB simulation are compared to reference data from an ANSYSFluent simulation in Figs. 13 and 14 As for previous cases, the LB model is able to capture the effects of varying specific heat capacity and thermal conductivity as a function of temperature. This is further illustrated by looking at the values of the $L_{2}$ and $L_{\infty}$ error norms on the center-lines. In the case of constant parameters, $L_{2}=4.568 \times 10^{-3}$ and $L_{\infty}=5.877 \times 10^{-3}$, while for variable parameters, $L_{2}=2.977 \times 10^{-3}$ and $L_{\infty}=3.987 \times 10^{-3}$. It is worth mentioning that for variable properties, the dynamic viscosity goes from 0.025

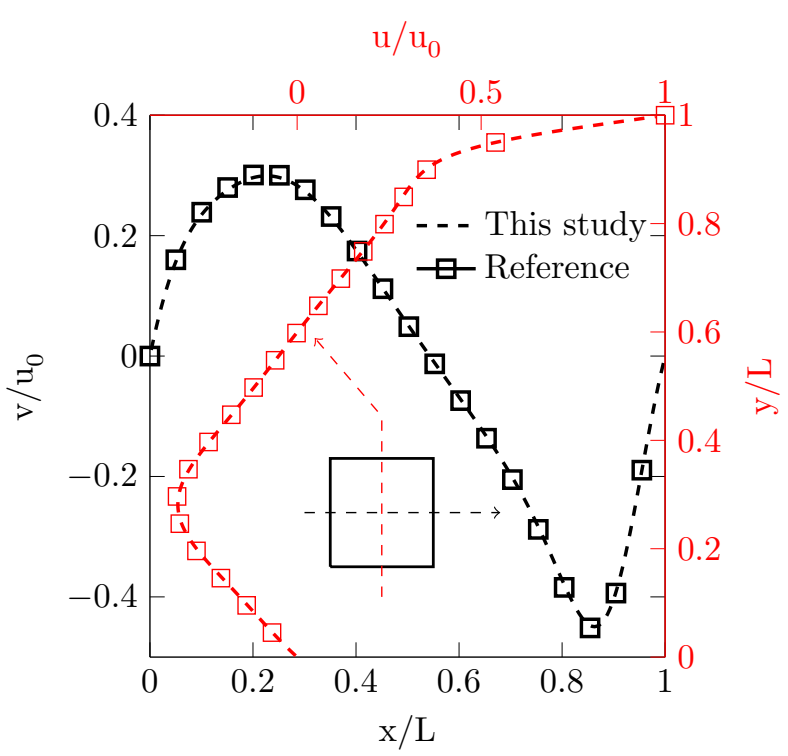

Figure 11: Constant properties velocity profiles along the vertical (red) and horizontal (black) center-lines for the heated lid-driven cavity

to $0.125 \mathrm{~kg} / \mathrm{m}$.s while $\lambda$ spans the interval between 0.125 and $0.6 \mathrm{~W} / \mathrm{m} . \mathrm{K}$ (which given the choices of $\delta_{t}, \delta_{x}$ and $\gamma$ results in $\tau=0.5375-0.68)$ and $c_{p}$ takes on values between 2.50 and $4.75 \mathrm{~J} / \mathrm{kg}$.K. As mentioned previously, cases such as this one, where specific heat capacity only changes with temperature, can also be modeled with the enthalpy scheme of Eqs. 36 to 40.

\subsection{Rectangular channel with mounted obstacles on upper and lower walls}

In order to confront the model to more realistic configurations, the case of a $2-\mathrm{D}$ channel flow with heated square obstacles mounted on both the upper and lower walls is considered [39, 40]. The geometrical configuration along with the boundary conditions are represented in Fig. 15. At the inlet (left) a Poiseuille velocity profile is imposed.

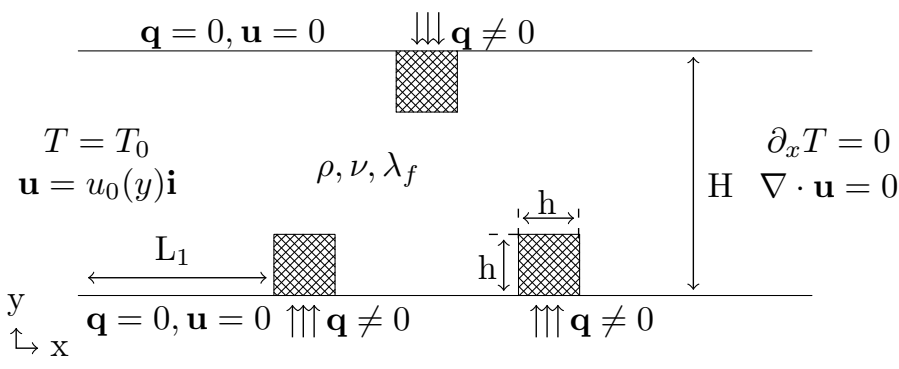

Figure 15: Configuration for rectangular channel flow with heated obstacles mounted on upper and lower walls

This configuration has been studied in details in 39, 40] at Reynolds numbers ranging from 400 to 2000 and considering different solid-to-fluid thermal conductivity ratios. 


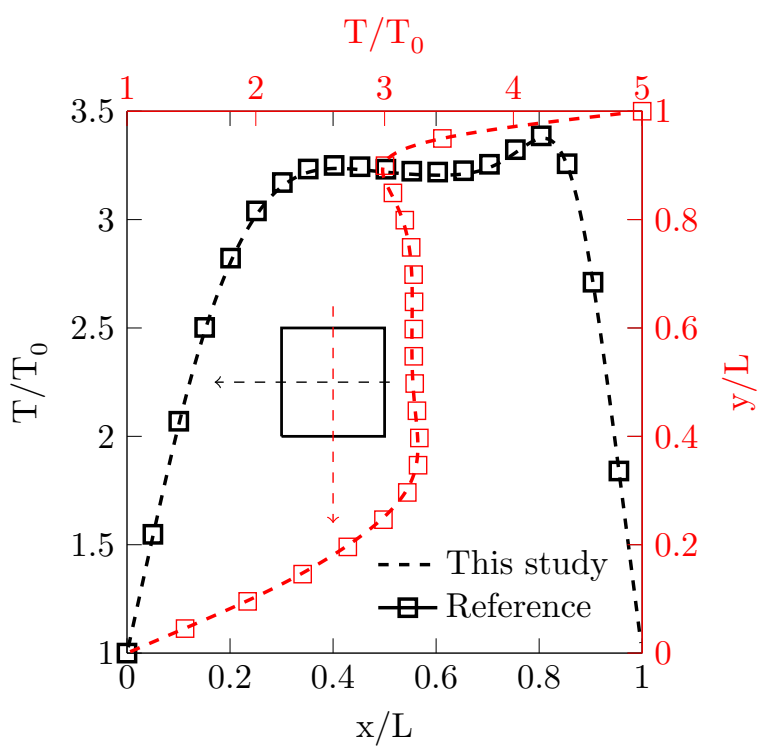

Figure 12: Constant properties temperature profiles along the vertical (red) and horizontal (black) center-lines for the heated lid-driven cavity

Here, a Reynolds number of 400 is considered, defined as:

$$
R e=\frac{D_{h} \bar{u}_{0}}{\nu}
$$

where $D_{h}$ is the hydraulic diameter defined as $D_{h}=2 H$ and $\bar{u}_{0}$ is the average inlet velocity. Following [39, 40], the Prandtl number is set to 0.71 and the solid-to-fluid thermal conductivity ratio, $\lambda_{s} / \lambda_{f}$ to 10 . The test-case being typical of electrical component cooling with air, the kinematic viscosity was taken as $1.568 \times 10^{-5} \mathrm{~m}^{2} / \mathrm{s}$, while thermal conductivity, density and specific heat capacity in the fluid were respectively set to $0.0262 \mathrm{~W} . \mathrm{m}^{-1} \mathrm{~K}^{-1}, 1.177$ $\mathrm{kg} / \mathrm{m}^{3}$ and 1004.9 J/kg.K. Following [39, 40, the density and specific heat capacity of the solid obstacle were set235 equal to that of the fluid. Furthermore, $H$ is set to $1 \mathrm{~mm}$ and $\bar{u}_{0}=3.136 \mathrm{~m} / \mathrm{s}$. The heat flux at the base of each obstacle, $q$ is fixed to $436.7 \mathrm{~W} \cdot \mathrm{m}^{-2}$.

Adiabatic, fixed-temperature, and fixed-velocity boundary conditions are applied using the previously mentioned formulations. The constant-flux boundary condition at the base of the solid obstacles is enforced following [41, where the missing populations are defined as:

$$
h_{\alpha}(\mathbf{x}, t)=\left(w_{\alpha}+w_{\bar{\alpha}}\right) T_{w}-h_{\bar{\alpha}}^{*}\left(\mathbf{x}, t-\delta_{t}\right)
$$

where $h_{\alpha}^{*}$ is the post-collision population and $T_{w}$ is defined as:

$$
T_{w}=\frac{2 q(\mathbf{x}, t)}{\lambda(\mathbf{x}, t)}+T\left(\mathbf{x}, t-\delta_{t}\right)
$$

In order to avoid interaction between the zero-gradient boundary conditions and the flow field, the first obstacle is placed at a distance of $L_{1}=5 H$ from the inlet while $L_{\text {out }}$, the horizontal distance between the last obstacle and the outlet boundary is set to $20 H$. The simulation is performed on a $80 \times 2100$ grid with $H=80$ and $h=20$. The

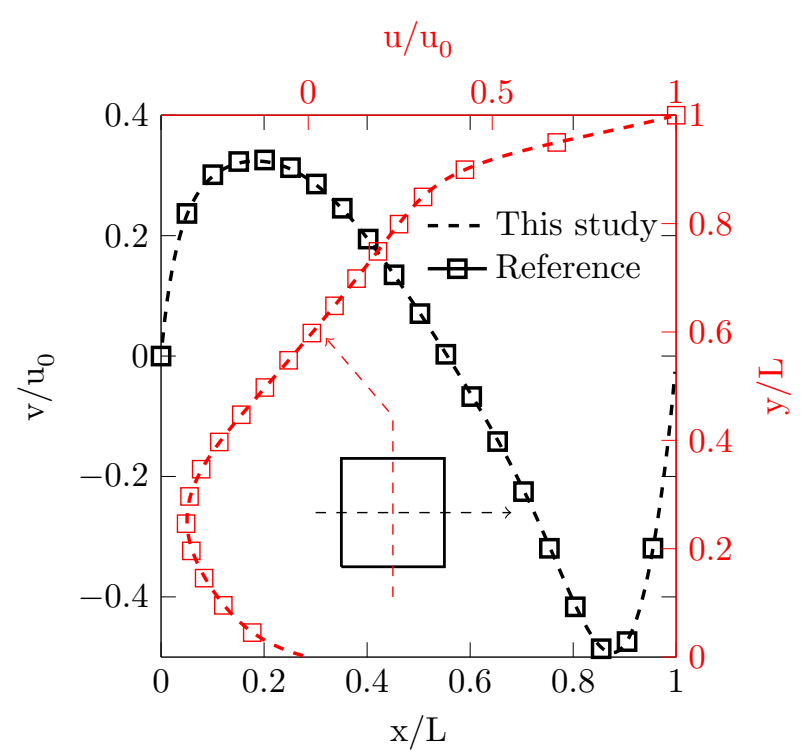

Figure 13: Variable properties velocity profiles along the vertical (red) and horizontal (black) center-lines for the heated lid-driven cavity

center-to-center horizontal distance between obstacles is $2 h$. The streamlines obtained at steady-state are shown in Fig. 16. In the context of this test-case $\delta_{x}$ is set to $2.5 \times 10^{-5} \mathrm{~m}$ and $\delta_{t}$ to $4.7 \times 10^{-8} \mathrm{~s}$ and $\gamma$ to 1 . This in turn results in $\tau_{H}=0.55-1$ and $\mathrm{Ma}=0.01$. Comparing the flow structure and recirculating zones to those reported in [39, 40, it is observed that they are in very good agreement. Furthermore, The non-dimensional temperature is shown in Fig. 17, defined as:

$$
\theta=\frac{T-T_{0}}{q H / \lambda_{f}} .
$$

The non-dimensional temperature iso-contours also agree very well with those reported in [39, 40. The non-dimensional temperature distributions on the surfaces of all obstacles are also compared to reference data from [39, 40] in Fig. 18 . 


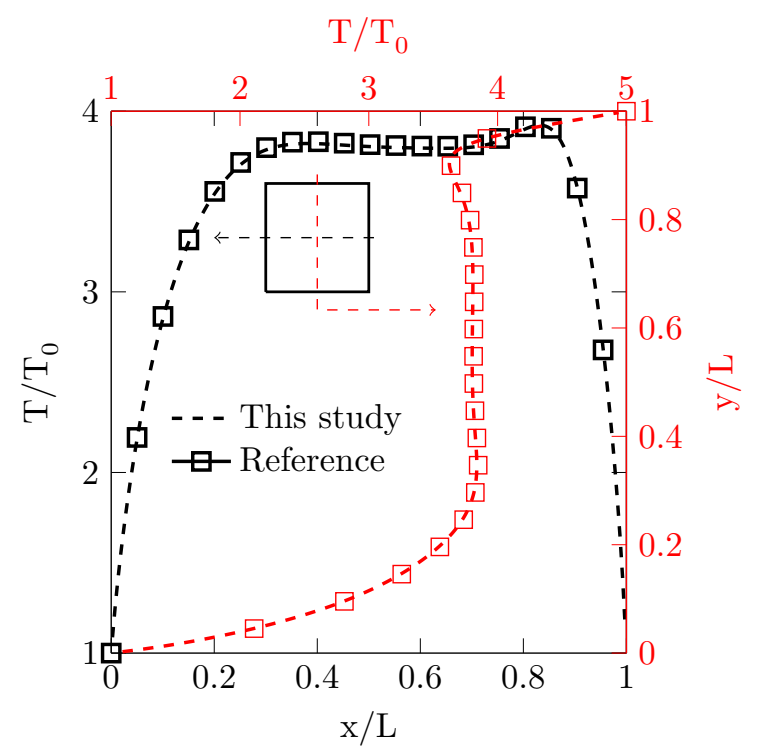

Figure 14: Variable properties temperature profiles along the vertical (red) and horizontal (black) center-lines for the heated lid-driven ${ }^{270}$ cavity with

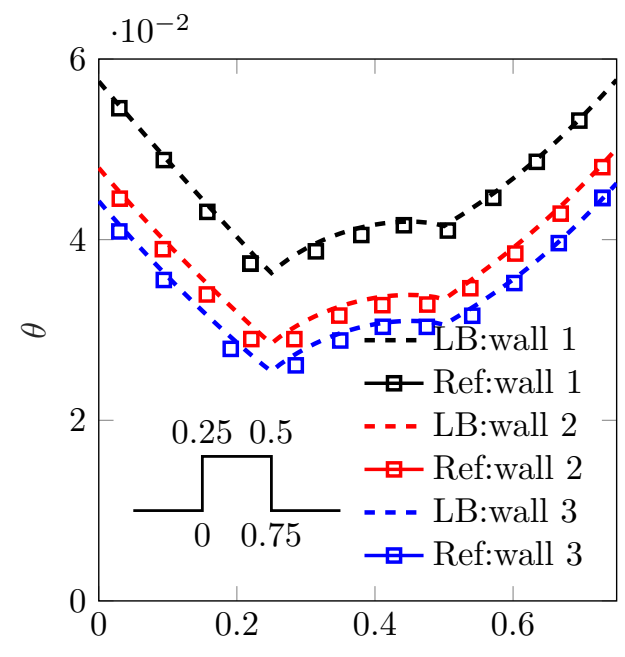

Obstacle peripheral distance

Figure 18: Distribution of non-dimensional temperature $\theta$ along the three faces of the heated obstacles at $\mathrm{Re}=400$

Comparing the non-dimensional temperature distribution as a function of the peripheral distance, starting from the left face and finishing on the right one, for each one of the three obstacles, it is observed that the proposed scheme is able to correctly model conjugate heat transfer even in complex flow configurations.

\subsection{Turbulent flow over a multi-layered wall-mounted cube}

The last configuration studied using the proposed model, is that of a heated wall-mounted cube subject to a turbulent flow field. In [42, the turbulent flow structure and surface heat transfer from a heated cube placed in a spa- of a plane channel was studied. To better understand the physics, a similar configuration was used to numerically study the case. In the numerical study, in order to reduce computation costs, instead of the whole channel and an array of cubes, a single heated cube of size $h$ was mounted on the center of the bottom wall. The overall geometrical configuration is shown in Fig. 19. To take into account flow field perturbation by the missing cube array, periodic boundary conditions were applied to the flow field in both $\mathrm{x}$ - and $\mathrm{y}$-directions. Given that in the original configuration none of the other cubes were heated, instead of periodic boundaries, constant temperature at $293.15 \mathrm{~K}$ and zero-gradient flux were respectively enforced at the inlet and outlet in the stream-wise direction [43]. The cube placed in the center of the bottom wall, in agreement with the experimental configuration, consists of a smaller constant temperature cube of size $0.8 \mathrm{~h}$ and temperature $348.15 \mathrm{~K}$ wrapped in a thin layer of epoxy of thickness $0.1 \mathrm{~h}$ [43. At the top and bottom walls zero-gradient boundary conditions were imposed on the temperature field. Following [43], the flow is studied at $\operatorname{Re}_{h}=\frac{h U_{0}}{\nu}=3854$ (corresponding to Reynolds number of 13,100 based on the channel height), where $U_{0}$ is the average velocity deduced from the imposed flow rate. The physical parameters of the working fluid -subscript f-and epoxy -subscript s-are given in Tables 3 and 4

\begin{tabular}{||c|c|c|c||}
\hline$\rho_{f}\left[\mathrm{~kg} / \mathrm{m}^{3}\right]$ & $\lambda_{f}[\mathrm{~W} / \mathrm{m} . \mathrm{K}]$ & $c_{p f}[\mathrm{~J} / \mathrm{kg} . \mathrm{K}]$ & $\nu\left[\mathrm{m}^{2} / \mathrm{s}\right]$ \\
\hline \hline 1.16 & 0.0257 & 1007.0 & $1.567 \times 10^{-5}$ \\
\hline
\end{tabular}

Table 3: Simulation parameters for unsteady three-block media testcase: fluid properties

\begin{tabular}{||c|c|c||}
\hline$\rho_{s}\left[\mathrm{~kg} / \mathrm{m}^{3}\right]$ & $\lambda_{s}[\mathrm{~W} / \mathrm{m} . \mathrm{K}]$ & $c_{p s}[\mathrm{~J} / \mathrm{kg} . \mathrm{K}]$ \\
\hline \hline 1150 & 0.236 & 1668.5 \\
\hline
\end{tabular}

Table 4: Simulation parameters for unsteady three-block media testcase: solid properties

The simulation has been performed on a $600 \times 600 \times 510$ grid, leading to $\delta_{x}=10^{-4} \mathrm{~m}$. The time-step $\delta_{t}$ was set to $2.48 \times 10^{-6} \mathrm{~s}$, leading to $\tau=0.51168$ and $\frac{\bar{U}_{0} \delta_{t}}{\delta_{x}}=0.1$. The flow field was modeled on a D3Q19 stencil while a D3Q7 stencil was used for the energy field. The simulation was initialized with a fluid at $T=293.15 \mathrm{~K}$. The simulation was carried out on the Neumann cluster at the "Ottovon-Guericke" university of Magdeburg, on 1400 processing units clocked at $2.4 \mathrm{GHz}$.

In order to maintain the flow at $\mathrm{Re}_{h}=3854$ during the simulation, a background constant pressure gradient in the stream-wise direction was added to the flow field. The additional pressure gradient $\nabla \cdot p_{0}(t)$ to apply at each timestep was defined as:

$$
\nabla \cdot p_{0}(t)=\left(\rho_{f} U_{0}-\rho \bar{u}_{x}(t)\right),
$$

where $\overline{u_{x}}$ is averaged over the entire domain at each timestep. According to [4], at the considered Reynolds num- 


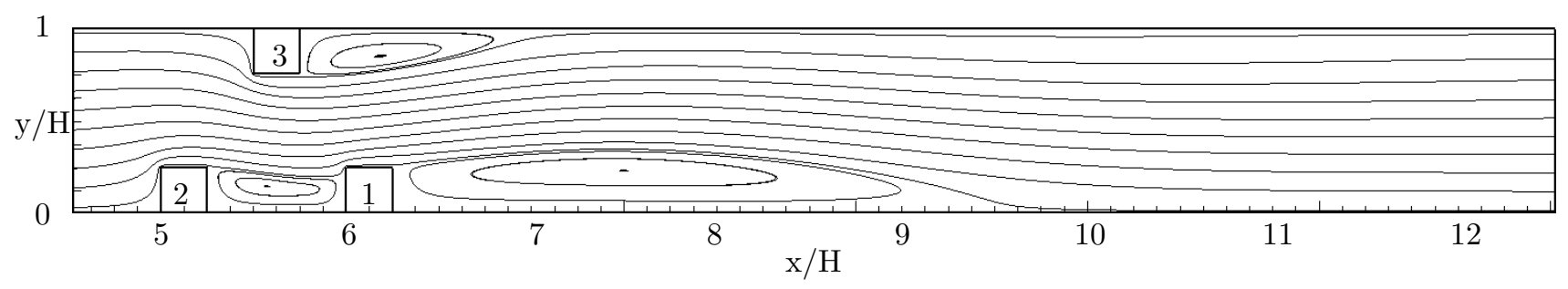

Figure 16: Flow structure for the channel flow with mounted obstacles at $\mathrm{Re}=400$

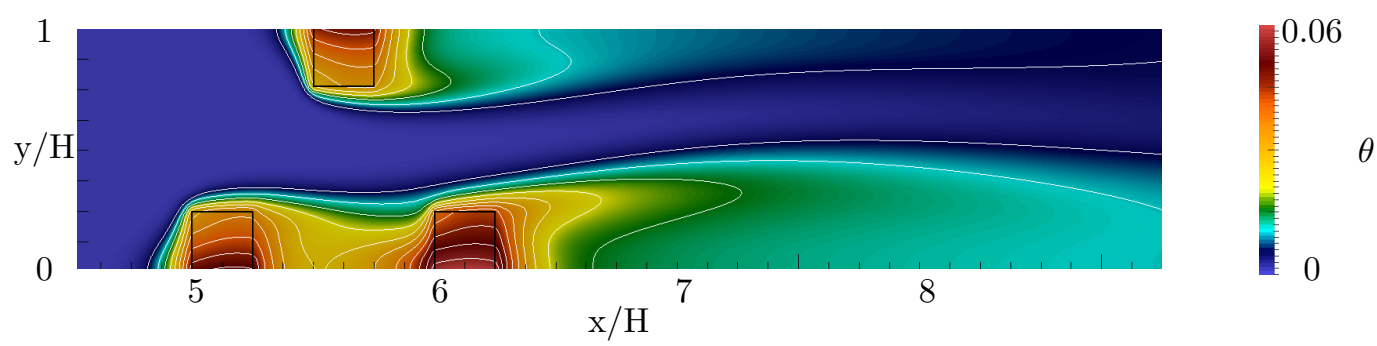

Figure 17: Non-dimensional temperature,$\theta$, iso-contours for the channel flow with mounted obstacles at Re $=400$

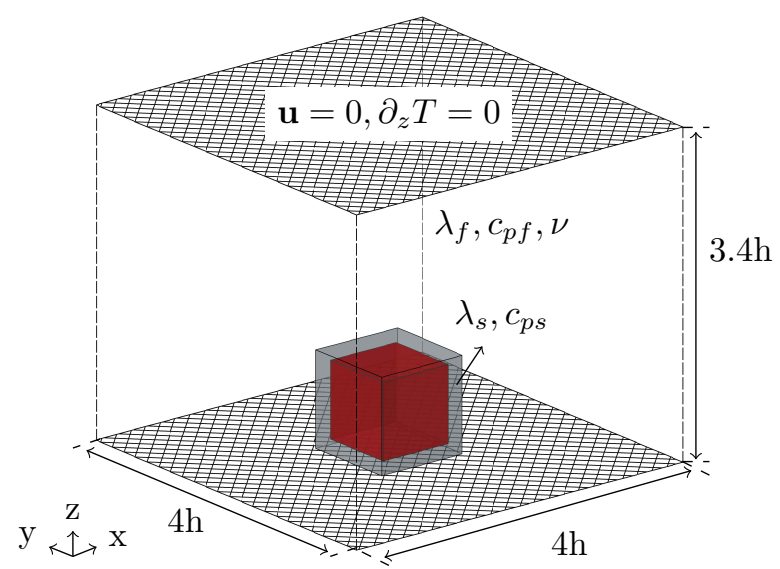

Figure 19: Geometrical configuration of multi-layered wall-mounted cube

ber, the dominant frequency is $f=27 \mathrm{~Hz}$, yielding a Strouhal number of $\mathrm{St}=0.109$ and a vortex shedding period of approximately 15000 time-steps.

In the context of this study, data averaging started after a transition period of 500,000 steps. The average fields reported in this section were taken over 2,000,000 steps. During the course of the transition period, the diffusion coefficient of the fluid, initially set to a higher value to295 accelerate convergence, was changed back to its original value after 50,000 steps 43].

The averaged vorticity iso-surface at three percent of the maximum vorticity is shown in Fig. 20.

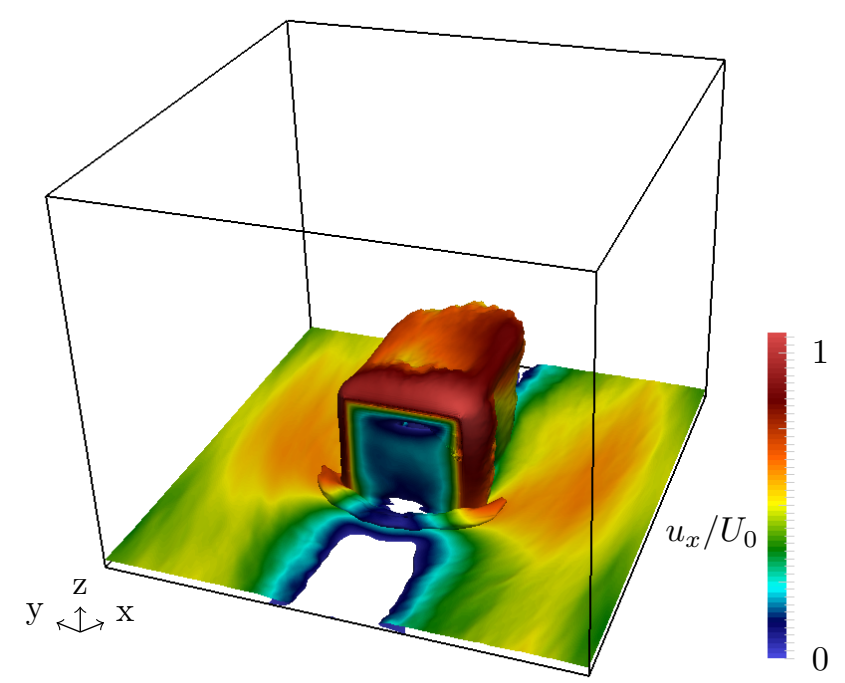

Figure 20: Iso-surface of the average vorticity magnitude. The isosurface value is fixed at $3 \%$ of the maximum value

Looking at Fig. 20, one can observe a large recirculating zone right in front of the cube in the form of a horseshoe. This observation is in agreement with reported results from both LES [4] and ELBM 26] simulations. The turbulent velocity field is further assessed through the average stream-wise velocity and the streamwise and spanwise diagonal components of the Reynolds stress tensor in Fig. 21. Two sets of reference data are shown alongside results from the present study in this figure: (a) Experimental data from 42 and LES results from 43. Overall, the results from the present study are in good agreement with the reference data. It is worth mentioning that the maximum non-dimensional velocity reached within the domain for this LB study, defined as $u_{\max } \delta_{t} / \delta_{x}$, was 0.15 ; 

non-dimensional velocities can affect the flow field in the form of compressibility error. Results from the present study are closer to experimental data compared to LES results from 43 in the core and near the upper wall. This might be attributed to the finer grid used for the present study in those regions. On the bottom wall however, near the recirculating zones both upstream and downstream of the obstacle, the LES results show better agreement with experimental data. This can be attributed to the grid clusfiner grids [43.

A quantitative analysis of the temperature field obtained through the present study is shown in Figs. 22 and 23 . In both figures, the obtained data is benchmarked against three sets of data: (a) experimental [42, (b) Large-Eddy ${ }^{350}$ Simulation 43 and (c) ELBM simulation [26]. In Fig. 22 . the temperature profile on the surface of the heated cube in the vertical plane located at $y / h=2$ is plotted. Figure 23 on the other hand shows the temperature profile on the cube surface in a horizontal plane located at $z / h=0.5$. The higher temperature observed near the bottom channel wall can be partly attributed to the zero-gradient boundary 355 condition applied to the energy solver there. This scheme only imposes zero flux in the direction perpendicular to the boundary, therefore allowing for heat transfer in the other directions along the wall surface. Overall, both figures show that the results obtained in the present study ${ }_{360}$ are within an acceptable range from previously reported data from either simulations or experiments.

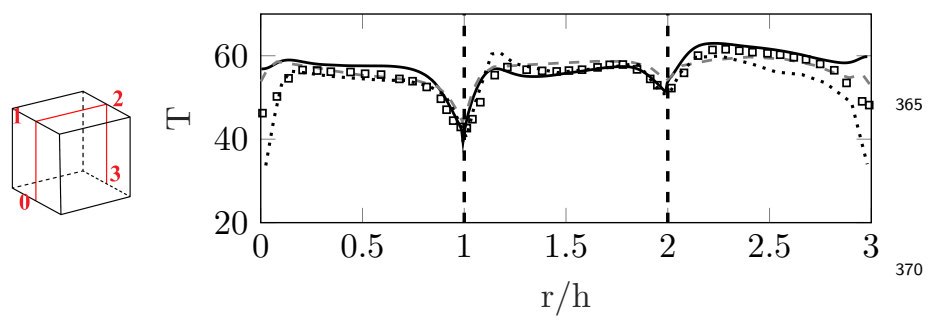

Figure 22: Surface temperature distribution along the 0-1-2-3 pathline on a vertical plane at $y / h=2$. Black solid line: present study, dashed gray line: LES [3], dotted black line: ELBM 26] and black ${ }^{375}$ square markers: experimental [42]

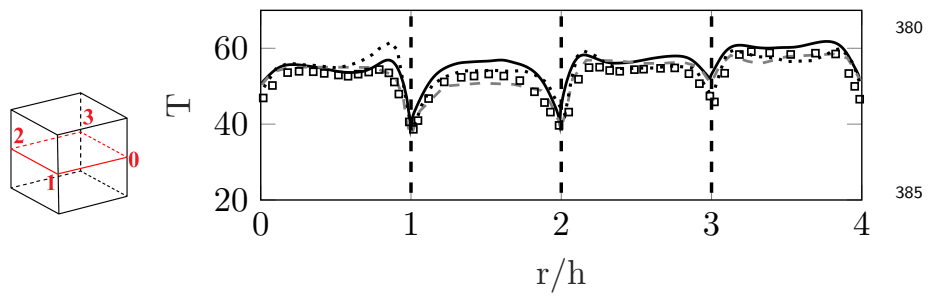

Figure 23: Surface temperature distribution along the $0-1-2-3-0390$ path-line on a horizontal plane at $\mathrm{z} / \mathrm{h}=0.5$. Black solid line: present study, dashed gray line: LES [43], dotted black line: ELBM [26] and black square markers: experimental 42 ]

\section{Conclusion}

Shortcomings of the classical advection-diffusion model for heat transfer were pointed out during the course of the present work. To overcome these issues, especially in the face of changes in thermo-physical properties, a modified model was proposed. It was shown that the proposed model can easily be extended to more complex configurations, e.g., multi-species and thermo-compressible flows. It can also take into account further effects such as viscous dissipation in an efficient way -their implementation will be the topic of future works.

The model was used to simulate a variety of test-cases, with temperature-dependent and spatially-varying properties. In all cases, the proposed model was able to correctly reproduce reference data.

The proposed model is currently being extended to multispecies flows, taking into account such effects as enthalpy transport through species diffusion.

\section{Acknowledgments}

The financial support of the German research foundation (DFG), Germany within the graduate college for micromacro-interactions in structured media and particle systems (GRK 1554) is gratefully acknowledged. The authors would also like to thank the reviewers for their pertinent remarks and insightful questions.

\section{References}

[1] S. Succi, The lattice Boltzmann equation: for fluid dynamics and beyond, Oxford university press, 2001.

[2] L. Zhong, S. Feng, P. Dong, S. Gao, Lattice Boltzmann schemes for the nonlinear Schrödinger equation, Physical Review E 74 (3) (2006) 036704.

[3] R. C. Coelho, A. S. Ilha, M. M. Doria, A lattice Boltzmann method based on generalized polynomials and its application for electrons in metals, EPL (Europhysics Letters) 116 (2) (2016) 20001.

[4] C. K. Aidun, J. R. Clausen, Lattice-Boltzmann method for complex flows, Annual review of fluid mechanics 42 (2010) 439-472.

[5] E. G. Flekkøy, Lattice Bhatnagar-Gross-Krook models for miscible fluids, Physical Review E 47 (6) (1993) 4247-4257.

[6] X. Shan, H. Chen, Lattice Boltzmann model for simulating flows with multiple phases and components, Physical Review E 47 (3) (1993) 1815.

[7] L.-S. Luo, S. S. Girimaji, Lattice Boltzmann model for binary mixtures, Physical Review E 66 (3) (2002) 035301.

[8] L.-S. Luo, S. S. Girimaji, Theory of the lattice Boltzmann method: two-fluid model for binary mixtures, Physical Review E 67 (3) (2003) 036302.

[9] X. He, S. Chen, G. D. Doolen, A novel thermal model for the Lattice Boltzmann method in incompressible limit, Journal of Computational Physics 146 (1) (1998) 282-300.

[10] F. J. Alexander, S. Chen, J. Sterling, Lattice Boltzmann thermohydrodynamics, Physical Review E 47 (4) (1993) R2249.

[11] Y. Qian, S. Orszag, Lattice BGK models for the Navier-Stokes equation: Nonlinear deviation in compressible regimes, EPL (Europhysics Letters) 21 (3) (1993) 255.

[12] X. Shan, Simulation of Rayleigh-bénard convection using a lattice Boltzmann method, Physical Review E 55 (3) (1997) 2780. 

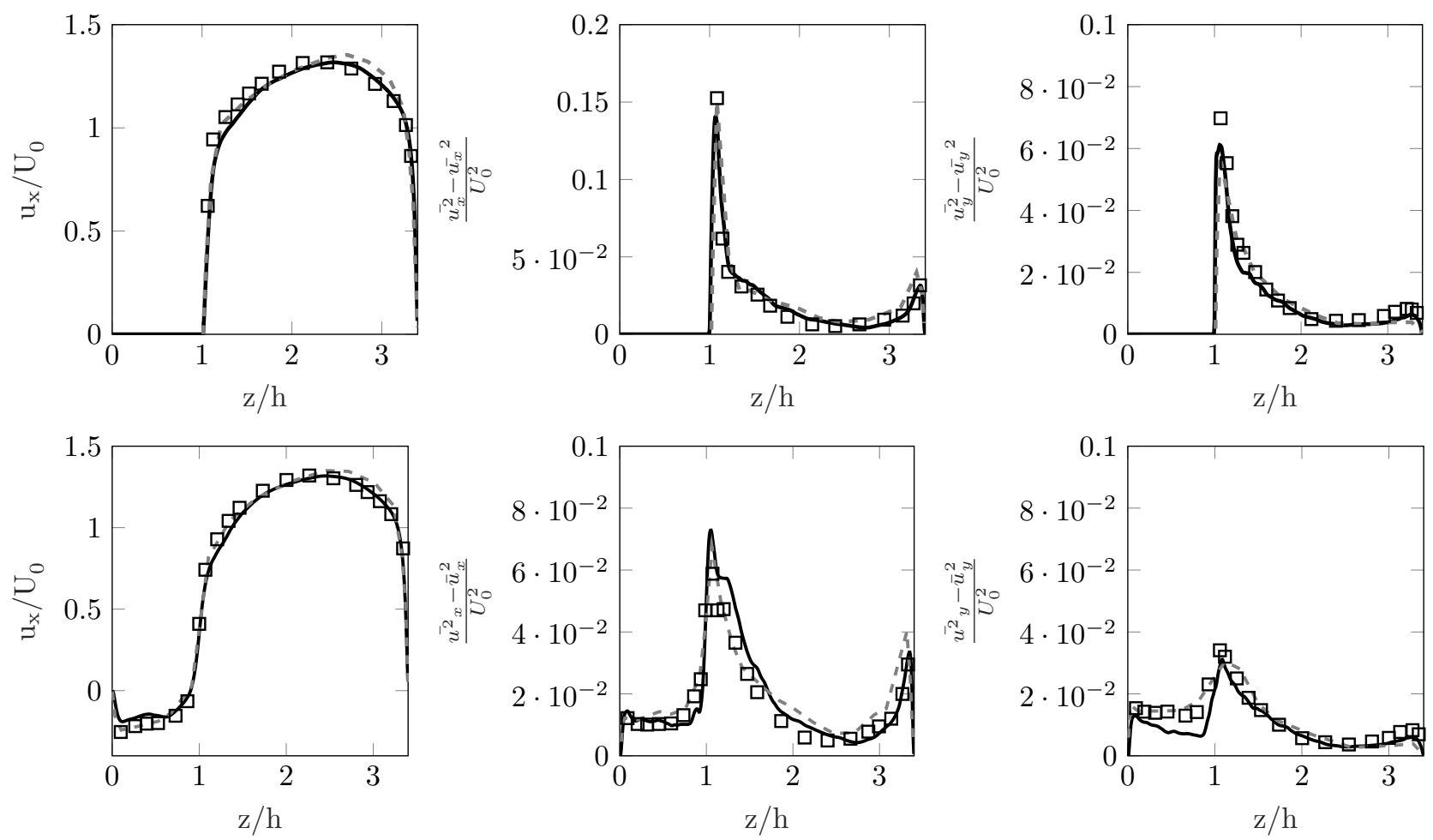

Figure 21: Normalized time-averaged turbulent velocity field profiles along two vertical lines on the vertical plane located at $y / h=2$. From top to bottom: $x / h=1.8$ and $x / h=2.8$. From left to right: average stream-wise velocity, stream-wise diagonal component of Reynolds stress tensor and span-wise diagonal component of Reynolds stress tensor respectively. Symbols: Black solid line: present study, dashed gray line: LES [43] and black square markers: experimental [4]

[13] Z. Guo, B. Shi, C. Zheng, A coupled lattice BGK model for the Boussinesq equations, International Journal for Numerical Methods in Fluids 39 (4) (2002) 325-342.

[14] Z. Guo, C. Zheng, B. Shi, T. Zhao, Thermal lattice Boltzmann430 equation for low Mach number flows: decoupling model, Physical Review E 75 (3) (2007) 036704.

[15] P. Lallemand, L.-S. Luo, Hybrid finite-difference thermal lattice boltzmann equation, International Journal of Modern Physics B 17 (01n02) (2003) 41-47.

[16] C. Obrecht, F. Kuznik, B. Tourancheau, J.-J. Roux, Multi-gpu implementation of a hybrid thermal lattice boltzmann solver using the thelma framework, Computers \& Fluids 80 (2013) 269-275.

[17] N. Prasianakis, K. Boulouchos, Lattice Boltzmann method for440 simulation of weakly compressible flows at arbitrary Prandtl number, International Journal of Modern Physics C 18 (04) (2007) 602-609.

[18] N. Frapolli, S. Chikatamarla, I. Karlin, Multispeed entropic lattice Boltzmann model for thermal flows, Physical Review E445 90 (4) (2014) 043306.

[19] N. Frapolli, S. S. Chikatamarla, I. Karlin, Simulations of heated bluff-bodies with the multi-speed entropic lattice Boltzmann method, Journal of Statistical Physics 161 (6) (2015) 1434-1452.

[20] N. Frapolli, S. S. Chikatamarla, I. V. Karlin, Entropic lattice450 Boltzmann model for compressible flows, Physical Review E 92 (6) (2015) 061301.

[21] S. A. Hosseini, A. Eshghinejadfard, N. Darabiha, D. Thévenin, Weakly compressible lattice boltzmann simulations of reacting flows with detailed thermo-chemical models, Computers \&455 Mathematics with Applications.

[22] M. Seddiq, M. Maerefat, M. Mirzaei, Modeling of heat transfer at the fluid-solid interface by lattice Boltzmann method, International Journal of Thermal Sciences 75 (2014) 28-35.

[23] A. Tarokh, A. Mohamad, L. Jiang, Simulation of conjugate heat 460 transfer using the lattice Boltzmann method, Numerical Heat Transfer, Part A: Applications 63 (3) (2013) 159-178.

[24] H. Karani, C. Huber, Lattice Boltzmann formulation for conjugate heat transfer in heterogeneous media, Physical Review E 91 (2) (2015) 023304

[25] Y. Hu, D. Li, S. Shu, X. Niu, Full eulerian lattice Boltzmann model for conjugate heat transfer, Physical Review E 92 (6) (2015) 063305.

[26] G. Pareschi, N. Frapolli, S. S. Chikatamarla, I. V. Karlin, Conjugate heat transfer with the entropic lattice boltzmann method, Physical Review E 94 (1) (2016) 013305.

[27] D. Gao, Z. Chen, L. Chen, D. Zhang, A modified lattice Boltzmann model for conjugate heat transfer in porous media, International Journal of Heat and Mass Transfer 105 (2017) 673-683.

[28] S. Chen, B. Yang, C. Zheng, A lattice Boltzmann model for heat transfer in heterogeneous media, International Journal of Heat and Mass Transfer 102 (2016) 637-644.

[29] T. Poinsot, D. Veynante, Theoretical and numerical combustion, RT Edwards Inc., 2005.

[30] B. Chopard, J. Falcone, J. Latt, The lattice Boltzmann advection-diffusion model revisited, The European Physical Journal Special Topics 171 (1) (2009) 245-249.

[31] R. Huang, H. Wu, Lattice Boltzmann model for the correct convection-diffusion equation with divergence-free velocity field, Physical Review E 91 (3) (2015) 033302.

[32] T. Krüger, H. Kusumaatmaja, A. Kuzmin, O. Shardt, G. Silva E. M. Viggen, The lattice boltzmann method, Springer International Publishing 10 (2017) 978-3.

[33] S. Hosseini, N. Darabiha, D. Thévenin, Mass-conserving advection-diffusion lattice Boltzmann model for multi-species reacting flows, Physica A: Statistical Mechanics and its Applications 499 (2018) 40-57.

[34] S. A. Hosseini, N. Darabiha, D. Thévenin, A. Eshghinejadfard, Stability limits of the single relaxation-time advection- 
diffusion lattice Boltzmann scheme, International Journal of Modern Physics C 28 (12) (2017) 1750141.

[35] J. Zhang, G. Yan, A lattice Boltzmann model for the reactiondiffusion equations with higher-order accuracy, Journal of Scientific Computing 52 (1) (2012) 1-16.

[36] Z. Chai, N. He, Z. Guo, B. Shi, Lattice Boltzmann model for high-order nonlinear partial differential equations, Physical Review E 97 (1) (2018) 013304.

[37] Z. Guo, C. Shu, Lattice Boltzmann method and its applications in engineering, Vol. 3, World Scientific, 2013.

[38] Y. Sun, I. S. Wichman, On transient heat conduction in a onedimensional composite slab, International Journal of Heat and Mass Transfer 47 (6-7) (2004) 1555-1559.

[39] A. Korichi, L. Oufer, Numerical heat transfer in a rectangular channel with mounted obstacles on upper and lower walls, International Journal of Thermal Sciences 44 (7) (2005) 644-655.

[40] A. Korichi, L. Oufer, Unsteady heat transfer and pressure drop in channels with obtacles mounted on the upper and lower walls, Numerical Heat Transfer, Part A: Applications 48 (7) (2005) $711-729$.

[41] T. Zhang, B. Shi, Z. Guo, Z. Chai, J. Lu, General bounceback scheme for concentration boundary condition in the latticeBoltzmann method, Physical Review E 85 (1) (2012) 016701.

[42] E. Meinders, K. Hanjalić, Vortex structure and heat transfer in turbulent flow over a wall-mounted matrix of cubes, International Journal of Heat and fluid flow 20 (3) (1999) 255-267.

[43] B. Ničeno, A. Dronkers, K. Hanjalić, Turbulent heat transfer from a multi-layered wall-mounted cube matrix: a large eddy simulation, International Journal of Heat and fluid flow 23 (2) (2002) 173-185.

\section{Appendix A. Multi-scale Chapman-Enskog expan- sion}

In this appendix, the asymptotic behavior of the discrete time-evolution equation of the proposed formulation will be analyzed through an approach that is now very common and well-established within the LB community. This approach consists of a Taylor-McLaurin expansion of the discrete operators followed by a multi-scale ChapmanEnskog expansion of the populations.

Starting from the discrete time evolution equation:

$$
\begin{aligned}
& h_{\alpha}\left(\mathbf{x}+\mathbf{c}_{\alpha} \delta_{t}, t+\delta_{t}\right)-h_{\alpha}(\mathbf{x}, t)= \\
& \frac{1}{\tau_{H}}\left(h_{\alpha}^{(e q)}(\mathbf{x}, t)-h_{\alpha}(\mathbf{x}, t)\right) \\
& \quad+w_{\alpha} \dot{\omega}_{T}(\mathbf{x}, t)+w_{\alpha} \mathbf{c}_{\alpha} \cdot \mathbf{F}(\mathbf{x}, t),
\end{aligned}
$$

Remembering the expressions for $h_{\alpha}^{(e q)}$ and $\mathbf{F}$ :

$$
\begin{array}{r}
h_{\alpha}^{(e q)}(\mathbf{x}, t)=w_{\alpha} H_{t}(\mathbf{x}, t)\left(1+\frac{1}{c_{s}^{2}} \mathbf{c}_{\alpha} \cdot \mathbf{u}(\mathbf{x}, t)\right) \\
+\eta_{\alpha}\left(\gamma T(\mathbf{x}, t)-H_{t}(\mathbf{x}, t)\right), \\
\mathbf{F}=\frac{1}{c_{s}^{2}}\left(1-\frac{1}{2 \tau_{H}}\right) \partial_{t}\left(H_{t} \mathbf{u}\right),
\end{array}
$$

and using a Taylor-Maclaurin expansion the LHS can be re-written as:

$$
\begin{aligned}
& h_{\alpha}\left(\mathbf{x}+\mathbf{c}_{\alpha} \delta_{t}, t+\delta_{t}\right)-h_{\alpha}(\mathbf{x}, t)= \\
& \delta_{t}\left(\partial_{t}+\mathbf{c}_{\alpha} \cdot \nabla\right) h_{\alpha}(\mathbf{x}, t)+\frac{\delta_{t}^{2}}{2}\left(\partial_{t}+\mathbf{c}_{\alpha} \cdot \nabla\right)^{2} h_{\alpha}(\mathbf{x}, t)+\mathcal{O}\left(\delta_{t}^{3}\right) .
\end{aligned}
$$

Re-writing the time-evolution equation in non-dimensional form using the following characteristic values:

$$
t^{+}=\frac{t}{T}, x^{+}=\frac{x}{L}, c^{+}=\frac{c}{L / T}
$$

one obtains:

$$
\begin{array}{r}
\delta_{t^{+}}\left(\partial_{t^{+}}+\mathbf{c}_{\alpha}^{+} \cdot \nabla^{+}\right) h_{\alpha}+\frac{\delta_{t^{+}}^{2}}{2}\left(\partial_{t^{+}}+\mathbf{c}_{\alpha}^{+} \cdot \nabla^{+}\right)^{2} h_{\alpha} \\
+\mathcal{O}\left(\delta_{t^{+}}^{3}\right)=\frac{1}{\tau_{H}}\left(h_{\alpha}^{(e q)}-h_{\alpha}\right) \\
\quad+w_{\alpha} \dot{\omega}_{T}+w_{\alpha} \mathbf{c}_{\alpha} \cdot \mathbf{F}
\end{array}
$$

In order to evaluate the macroscopic behavior of the scheme the following multi-scale expansions are introduced:

$$
\begin{gathered}
h_{\alpha}=h_{\alpha}^{(0)}+\epsilon h_{\alpha}^{(1)}+\epsilon^{2} h_{\alpha}^{(2)}+\mathcal{O}\left(\epsilon^{3}\right), \\
\delta_{t^{+}} \partial_{t^{+}}=\epsilon \partial_{t^{+}}^{(1)}+\epsilon^{2} \partial_{t^{+}}^{(2)}+\mathcal{O}\left(\epsilon^{3}\right), \\
\delta_{t^{+}} \nabla^{+}=\epsilon \nabla^{+,(1)}, \\
\mathbf{F}^{+}=\epsilon \mathbf{F}^{+,(1)}+\epsilon \mathbf{F}^{+,(2)}+\mathcal{O}\left(\epsilon^{3}\right), \\
\dot{\omega}_{T}^{+}=\epsilon^{2} \dot{\omega}_{T}^{+,(2)}+\mathcal{O}\left(\epsilon^{3}\right),
\end{gathered}
$$

where:

$$
\forall i \neq 0, \sum_{\alpha} h_{\alpha}^{(i)}=0 .
$$

which lead to the following equations at different orders in $\epsilon$ :

$$
\epsilon^{0}: h_{\alpha}^{(e q)}=h_{\alpha}^{(0)} \text {, }
$$

$$
\begin{gathered}
\epsilon^{1}: D_{t^{+}}^{(1)} h_{\alpha}^{(0)}=-\frac{1}{\tau_{H}} h_{\alpha}^{(1)}+w_{\alpha} \mathbf{c}_{\alpha}^{+} \cdot \mathbf{F}^{+,(1)} \\
\epsilon^{2}: \partial_{t^{+}}^{(2)} h_{\alpha}^{(0)}+D_{t^{+}}^{(1)} h_{\alpha}^{(1)}+\frac{1}{2} D_{t^{+}}^{(1)} h_{\alpha}^{(0)}= \\
-\frac{1}{\tau_{H}} h_{\alpha}^{(2)}+w_{\alpha} \mathbf{c}_{\alpha}^{+} \cdot \mathbf{F}^{+,(2)}+w_{\alpha} \dot{\omega}_{T}^{+,(2)}
\end{gathered}
$$

where $D_{t^{+}}^{(1)}=\partial_{t^{+}}^{(1)}+\mathbf{c}_{\alpha}^{+} \cdot \nabla^{+,(1)}$. Taking the zeroth-order moment of Eq. A.13, the following conservation equation is recovered at first order in $\epsilon$ :

$$
\epsilon^{1}:\left(\partial_{t^{+}}^{(1)}+\nabla^{+,(1)} \cdot \mathbf{u}^{+}\right) H_{t}=0 .
$$


Up to this point, the new set of weight functions $\eta_{\alpha}$ does not intervene as it does not have zeroth and first-order moments. In Eq. A.15. multiplying Eq. A.14 by $\frac{\epsilon}{2} D_{t^{+}}^{(1)}$, the last term on the LHS can be replaced by the following expression:

$$
\epsilon^{2}: \frac{1}{2} D_{t^{+}}^{(1)^{2}} h_{\alpha}^{(0)}=-D_{t^{+}}^{(1)} \frac{1}{2 \tau_{T}} h_{\alpha}^{(1)}+D_{t^{+}}^{(1)} \frac{w_{\alpha}}{2} \mathbf{c}_{\alpha}^{+} \cdot \mathbf{F}^{+,(1)}
$$

Introducing this expression back into Eq. A.15.

$$
\begin{aligned}
& \epsilon^{2}: \partial_{t^{+}}^{(2)} h_{\alpha}^{(0)}+D_{t^{+}}^{(1)}\left(1-\frac{1}{2 \tau_{H}}\right) h_{\alpha}^{(1)} \\
&+D_{t^{+}}^{(1)} \frac{w_{\alpha}}{2} \mathbf{c}_{\alpha}^{+} \cdot \mathbf{F}^{+,(1)}=-\frac{1}{\tau_{H}} h_{\alpha}^{(2)}+w_{\alpha} \mathbf{c}_{\alpha}^{+} \cdot \mathbf{F}^{+,(2)} \\
&+w_{\alpha} \dot{\omega}_{T}^{+,(2)},
\end{aligned}
$$

then replacing $h_{\alpha}^{(1)}$ with its expression from Eq. A.14

$$
\begin{aligned}
& \epsilon^{2}: \partial_{t^{+}}^{(2)} h_{\alpha}^{(0)}+D_{t^{+}}^{(1)}\left(\frac{1}{2}-\tau_{H}\right) D_{t^{+}}^{(1)} h_{\alpha}^{(0)} \\
& +D_{t^{+}}^{(1)} \tau_{H} w_{\alpha} \mathbf{c}_{\alpha}^{+} \cdot \mathbf{F}^{+,(1)}=-\frac{1}{\tau_{H}} h_{\alpha}^{(2)}+w_{\alpha} \mathbf{c}_{\alpha}^{+} \cdot \mathbf{F}^{+,(2)} \\
& +w_{\alpha} \dot{\omega}_{T}^{+,(2)},
\end{aligned}
$$

and taking the zeroth-order moment:

$$
\begin{aligned}
\epsilon^{2}: \partial_{t^{+}}^{(2)} H_{t}-\nabla^{+,(1)} \cdot \frac{2 \tau_{T}-1}{2} c_{s}^{2} \gamma \nabla^{+,(1)} \cdot T \mathbf{I} & \\
-\dot{\omega}_{T}^{+,(2)} & =0 .
\end{aligned}
$$

The second term on the LHS is obtained by computing the second-order moment of the EDF:

$$
\sum_{\alpha} \mathbf{c}_{\alpha} \otimes \mathbf{c}_{\alpha} h_{\alpha}^{(e q)}=c_{s}^{2} \gamma T \mathbf{I}
$$

without the second part of EDF with the second set of weight functions, we would have had:

$$
\sum_{\alpha} \mathbf{c}_{\alpha} \otimes \mathbf{c}_{\alpha} h_{\alpha}^{(e q)}=c_{s}^{2} H_{t} \mathbf{I}
$$

Adding up Eqs. A.16 and A.20, and making use of the nondimensionalization equations introduced in the beginning of this section the following equation is obtained:

$$
\begin{aligned}
\partial_{t} H_{t} & +\nabla \cdot\left(H_{t} \mathbf{u}\right) \\
& -\nabla \cdot\left[\frac{c_{s}^{2} \gamma \delta_{t}\left(2 \tau_{H}-1\right)}{2} \nabla \cdot(T \mathbf{I})\right]-\dot{\omega}_{T}=0
\end{aligned}
$$

Therefore, to get the correct macroscopic equation, the relaxation coefficient must be set to:

$$
\lambda=\frac{c_{s}^{2} \delta_{t} \gamma\left(2 \tau_{H}-1\right)}{2}
$$

